\title{
Erratum to "Slow Open Dialectical Behavior Group Therapy for Adolescents and Parents: Longitudinal Study of Unconscious Orientation" [Psychology 12 (2021) 756-784]
}

\author{
Aranzazu Fernandez-Rivas1,2, Eva Sesma-Pardo1,2, Iñaki Kerexeta1,2, Aida Diaz-Cosgaya1, \\ Esther Vivanco', Federico Carminati ${ }^{3,4}$, Miguel Angel Gonzalez Torres ${ }^{1,2}$, Claire Fouassier ${ }^{3,4}$, \\ François Martin ${ }^{5}$, Jacques Demongeot ${ }^{6}$, Giuliana Galli Carminati ${ }^{3,4,7}$ \\ ${ }^{1}$ Psychiatry Service, Basurto University Hospital-Osakidetza, Bilbao, Spain \\ ${ }^{2}$ Neurosciences Department, Basque Country University, Bilbao, Spain \\ ${ }^{3}$ ASsociation Pour le TRAvail Groupal Therapeutique et Social (ASTRAG) and Simposietto, Geneva, Switzerland \\ ${ }^{4}$ Société Internationale de Psychanalyse Multidisciplinaire (SIPsyM), Geneva, Switzerland \\ ${ }^{5}$ CNRS, Paris, France \\ ${ }^{6}$ Faculté de Médecine, Université Grenoble Alpes, La Tronche, France \\ ${ }^{7}$ SNUBH (Seoul National University, Bundang Hospital) eoul National University (Bundang Hospital), Seoul, Republic of Korea \\ Email: giuliana.gallicarminati@gmail.com
}

How to cite this paper: Fernandez-Rivas, A., Sesma-Pardo, E., Kerexeta, I., DiazCosgaya, A., Vivanco, E., Carminati, F., Torres, M. A. G., Fouassier, C., Martin, F., Demongeot, J., \& Carminati, G. G. (2021). Erratum to "Slow Open Dialectical Behavior Group Therapy for Adolescents and Parents: Longitudinal Study of Unconscious Orientation" [Psychology 12 (2021) 756-784]. Psychology, 12, 1615-1641.

https://doi.org/10.4236/psych.2021.1210100

Received: September 30, 2021

Accepted: October 24, 2021

Published: October 27, 2021

Copyright $\odot 2021$ by author(s) and Scientific Research Publishing Inc. This work is licensed under the Creative Commons Attribution International License (CC BY 4.0). http://creativecommons.org/licenses/by/4.0/

\begin{abstract}
The original online version of this article (Fernandez-Rivas, A., Sesma-Pardo, E., Kerexeta, I., Diaz-Cosgaya, A., Vivanco, E., Carminati, F., Torres, M., Fouassier, C., Martin, F., Demongeot, J. and Carminati, G. (2021) Slow Open Dialectical Behavior Group Therapy for Adolescents and Parents: Longitudinal Study of Unconscious Orientation. Psychology, 12, 756-784. doi: https://doi.org/10.4236/psych.2021.125047) was published in Vol.12 No.5, May 2021. The author wishes to correct the errors to the following content.
\end{abstract}

\section{Keywords}

Erratum

\section{Introduction}

This work continues our investigation in the possibility of quantifying or, at the least, objectifying group dynamics (Fernandez-Rivas et al., 2020; Trojaola-Zapirain et al., 2014, 2015, 2016, 2019). We have already described the basic principles that have led us to initiate this work in the referenced works, and therefore we 
will give here only a short reminder. The study's objective is to explore the existence and the evolution of common behavior of the participants to four slow open groups taking part in a Dialectical Behavior Therapy (DBT) skills training. Two groups were composed of adolescents and two of their respective parents. We analyzed their evolution via the answers to the "absurd" questionnaire.

Dialectical Behavioral Therapy (DBT) (Linehan, 1993, 2015; Miller et al., 2017) has demonstrated an effective transdiagnostic treatment for adolescents whose main symptoms are emotional dysregulation and impulsivity. A complete program based on this therapy has been implemented in the Psychiatric Service of Basurto University Hospital (Bilbao, Spain). This therapeutic system involves several modules, including DBT skills groups for adolescents and parents.

According to Bion, there are universal principles-that he calls "basic assumptions"- guiding the group's behavior and its members in continual interaction with the external and internal realities of the group itself (Bion, 1961; Foulkes, 1964; Vergopoulos, 1983). This theory has led some psychoanalysts to suppose a "group" nature of the individual psyche (Kaës, 2010).

Bion also formulated the hypothesis of the existence of a group psychical apparatus. This hypothesis means that when the "basic assumptions" govern the group's behavior, we cannot consider the group members as separated entities but rather as the expression of a single psychical entity (Bion, 1961). It is intriguing to observe that this occurs for interacting quantum objects forming an entangled state (Aspect et al., 1982; Bell, 1964, 1966; Bohr, 1935; Einstein et al., 1935; Richens et al., 2017; Schrödinger, 1935, 1936).

Although never really interested in the study of the "collective", from which, according to him, the individual has to emerge via the process of individuation, Carl Gustav Jung has nevertheless provided one of the most potent metaphors for the "collective soul". According to him (Jung \& McGuire, 1925), the individual psyche lies upon a stratified unconscious in which each successive layer belongs to a broader community of people and even, for the most profound strata, living beings. This "collective unconscious" (Jung, 1959) is the "locus" of the connections between the world of reality and the world of the soul. These connections appear "acausal" but, according to Jung (Jung, 1952, 1960), respond to the unfathomable logic of the unknowable collective unconscious.

The emergence of these connections into the sensible world gives rise to what Jung termed "synchronicity" (Jung, 1952), the meaningful correlation between an inner condition-feeling or thought—and an external event with no apparent causal connection.

When Jung met W. Pauli, one of the most profound and prolific physicists of the $20^{\text {th }}$ century, the concept of synchronicity was connected to the newly discovered phenomenon of quantum entanglement that will become a fundamental-albeit controversial-cornerstone of the new physics of quantum phenomena. This gave rise to a life-long intellectual collaboration between Jung and Pauli in pursuit of the dream to root both the description of the material world 
and that of the soul in the same basic principles (Jung et al., 2001).

Humbly following in their footsteps, we have described the interaction between the unconscious in terms of quantum entanglement. This description supposes the existence of a universal quantum field of (un)consciousness (Baaquie \& Martin, 2005; Orlov, 1982) relating to all living creatures. Therefore, two interacting individuals would temporarily lose their individuality and form a connected system during their interaction. (Galli Carminati et al., 2017; Galli Carminati \& Martin, 2008; Martin et al., 2009, 2010, 2013). It is natural to extend such a model to a group of individuals (Grinberg-Zylberbaum et al., 1994; Martin \& Galli Carminati, 2009), where the entanglement between the different unconscious can bring to the formation of a single entity with a distinct behavior, explaining the correlations observed between group members (Marshall, 1989). These developments are part of a larger discipline known as psychophysics (Beck \& Eccles, 1992; Conte et al., 2003; Freeman \& Vitiello, 2016; Hameroff \& Penrose, 1996; Penrose \& Gardner, 1989; Penrose, 1994; Pitkänen, 1998, 2012; Sabbadini \& Vitiello, 2019; Vitiello, 2003; Zurek, 1981).

In a recent series of works (Galli Carminati \& Carminati, 2006; Galli Carminati \& Martin, 2008; Martin, Carminati, \& Galli Carminati, 2009; Martin, Carminati, \& Galli Carminati, 2010; Martin, Carminati, \& Galli Carminati, 2013), some of the authors of the present paper have focused on the possibility to describe the group psyche with concepts and models borrowed from Quantum Mechanics.

Some of the authors of the present work have formulated the hypothesis that, following the basic tenets of psychophysics, also the group psyche could be described by a multi-body entangled system (Galli Carminati \& Martin, 2008; Martin et al., 2009, 2010, 2013; Martin \& Galli Carminati, 2009). Moreover, this hypothesis has led to the formulation of the "absurd experiment" that is the subject of this paper, which has already been performed on different psychodynamics groups (Fernandez-Rivas et al., 2020; Trojaola-Zapirain et al., 2014, 2015, 2016, 2019).

Although the concept of entanglement of the unconscious is not new (Atmanspacher et al., 2002; Atmanspacher \& Fach, 2013; Fach, 2011; Khrennikov, 2015; Walach \& Römer, 2011), we failed to find in the literature an experiment similar to the one presented in this paper, which seems to be therefore entirely original.

The rationale of conducting this experiment is to try to quantify, or at least objectify, one aspect of the unconscious, measuring its effect on the participants' behavior in this study. The authors have made the hypothesis that the group environment could "amplify" a "microscopic" entanglement between the unconscious to render it detectable at the macroscopic level. The present experiment is similar to the previous ones conducted by the same authors on training groups (Trojaola-Zapirain et al., 2014, 2015, 2016, 2019) and DBT closed groups (Fernandez-Rivas et al., 2020). In this latter study, we have introduced a "questionnaire zero" to evaluate the socio-cultural bias in the choices before the groups 
meet. The novelty of the present study is that it uses the same experimental setup on two "slow open" groups of adolescents and their parents who were following a Dialectical Behavior Therapy (DBT) skills training at the Psychiatric Service of Basurto University Hospital in Bilbao, Spain.

This setup is different from the ones considered before since we cannot any longer consider a "closed" system, but rather two couples of systems interacting with each other and, necessarily, open to the environmental and existential situations and influences of the larger social group.

The perspective of this study is to observe the evolution of the answers to the questionnaire, considering whether the group dynamics is also tributary of the larger social group interacting as the environment in quantum mechanics.

\section{Materials and Methods}

\subsection{Participants}

We have included in this study four different groups of participants: two composed of adolescents participating in Dialectical Behavior Therapy (DBT) skills training and two others consisting of their respective parents, also participating in DBT skills training. The demography of the groups is presented in Table 1.

The adolescents followed two slow-open DBT skills training groups of 2-hour sessions every week. One of the participants was excluded from the study because she did not participate in any group sessions. The group of parents was a slow open group of 10 DBT sessions of 1.5 hours each, also weekly.

The study started after the Basurto University Hospital Ethics Committee (Bilbao, Spain) gave its approval in adherence to the Helsinki Declaration for research with human subjects. All participants gave written informed consent after receiving oral and written information about the experiment, and specifically for adolescents, both the participant and their parents or legal tutor signed

Table 1. Demographic, social, and group composition of the participant sample with the age of participants in each group, adolescent and member of their family.

\begin{tabular}{|c|c|c|c|c|}
\hline & Adolescent G1 & Adolescent G2 & Parents G1 & Parents G2 \\
\hline Total & 21 & 16 & 25 & 19 \\
\hline Female & $14(66.7 \%)$ & $14(87.5 \%)$ & $18(72 \%)$ & $11(57.9 \%)$ \\
\hline Average age & 15.6 & 16.4 & & \\
\hline $1 Q-3 Q$ & $14.9-16.5$ & $15.3-17.4$ & & \\
\hline Biological Family & 8 & 7 & & \\
\hline Adoptive Family & 1 & 2 & & \\
\hline Single Parent & 10 & 6 & & \\
\hline Other living situations & 2 & 1 & & \\
\hline Undergrad education & 17 & 9 & & \\
\hline Graduate education & 4 & 6 & & \\
\hline Postgraduate education & & 1 & & \\
\hline
\end{tabular}


informed consents. All participant data were coded to be completely anonymous, including for the researchers analyzing the data.

\subsection{Procedure}

The skills taught in these groups are mindfulness, distress tolerance, regulation of emotions, interpersonal effectiveness, and walking the middle path. The group for adolescents consists of 162 -hour sessions weekly. And the group for parents consists of 10 sessions of 1.5 hours duration, also weekly.

The groups included in this study were "slow-open," which means that participants could enter and leave the group during the training. Two therapists ran each group.

Before joining the group, all participants (adolescents and parents) attended an interview for evaluation and information on the group's methodology and the research. During that interview, participants filled the informed consent and the questionnaire number "zero" (with one exception). In addition, sociodemographic data on adolescents were collected (see Table 1).

An identification code was assigned to each participant to keep his identity anonymous for the research. We have extensively described the general setting of this experiment in previous publications (Fernandez-Rivas et al., 2020; Trojaola-Zapirain et al., 2014, 2015, 2016, 2019). It will be enough to recall that we have used a questionnaire composed of 50 pairs of figures for this work. Participants were asked to select one picture from each pair and to complete the questionnaire in three minutes. The pictures' choice aims to minimize the socio-cultural bias introduced by a word questionnaire (Zanello et al., 2004). The figures in each pair were always the same, but the pairs were randomly reshuffled at each test repetition to minimize mnemonic effects. Figure 1 reports a sample page from the questionnaire with fictional picture choices.

We will name the four groups PG1 (first parent group), PG2 (second parent group), YG1 (first adolescent group), and YG2 (second adolescent group). Not all the parents participated in the groups. The duration of the four training
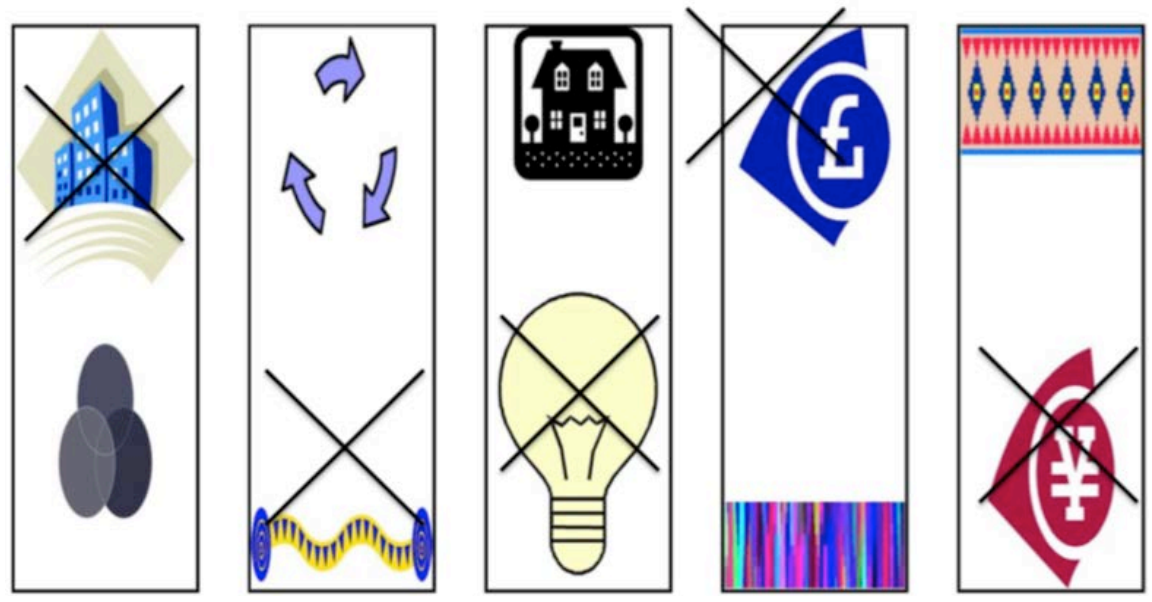

Figure 1. A page from the questionnaire with "fake" answers. 
courses is, respectively, 43, 31, 43, and 27 sessions.

The participation has been somewhat uneven to the different groups (see Figures 2-4). The presence in the group of adolescents was less regular after the $5^{\text {th }}$ session.

\section{Number of participants in the groups}

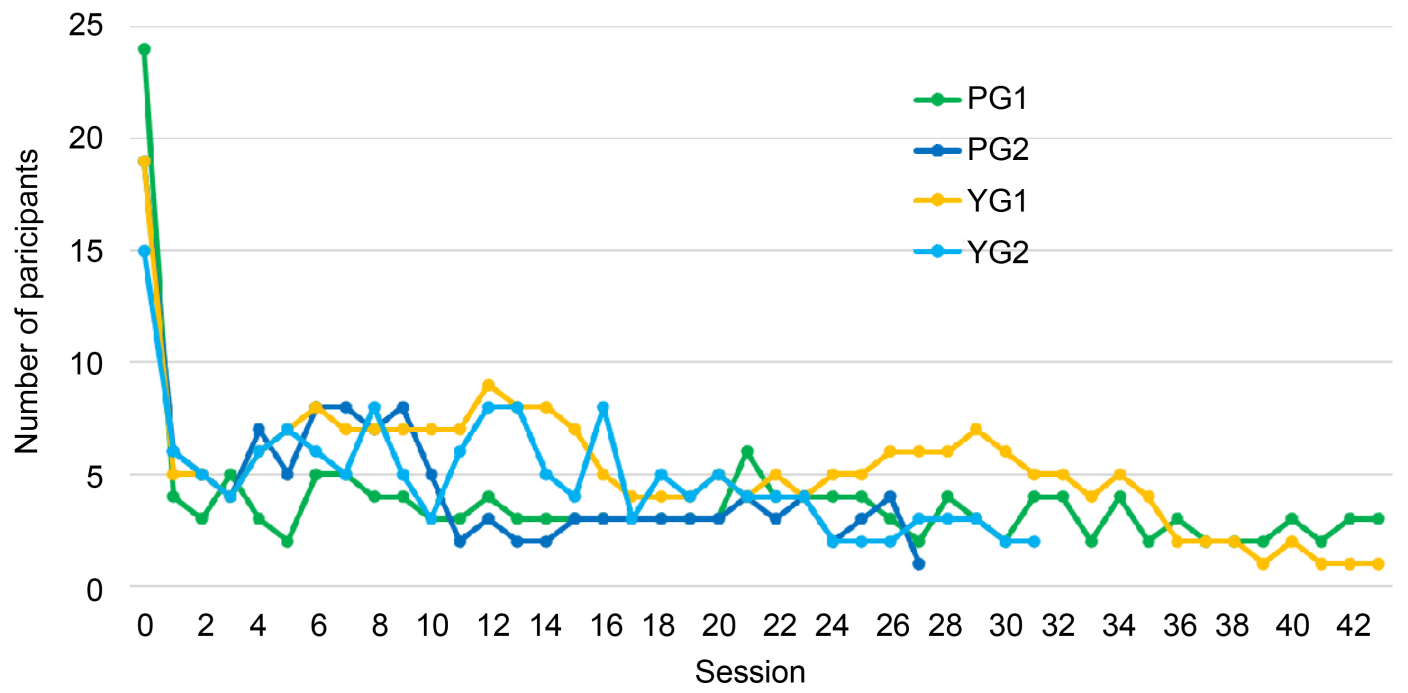

Figure 2. Number of participants in the different sessions.

Participation to the differrent sessions

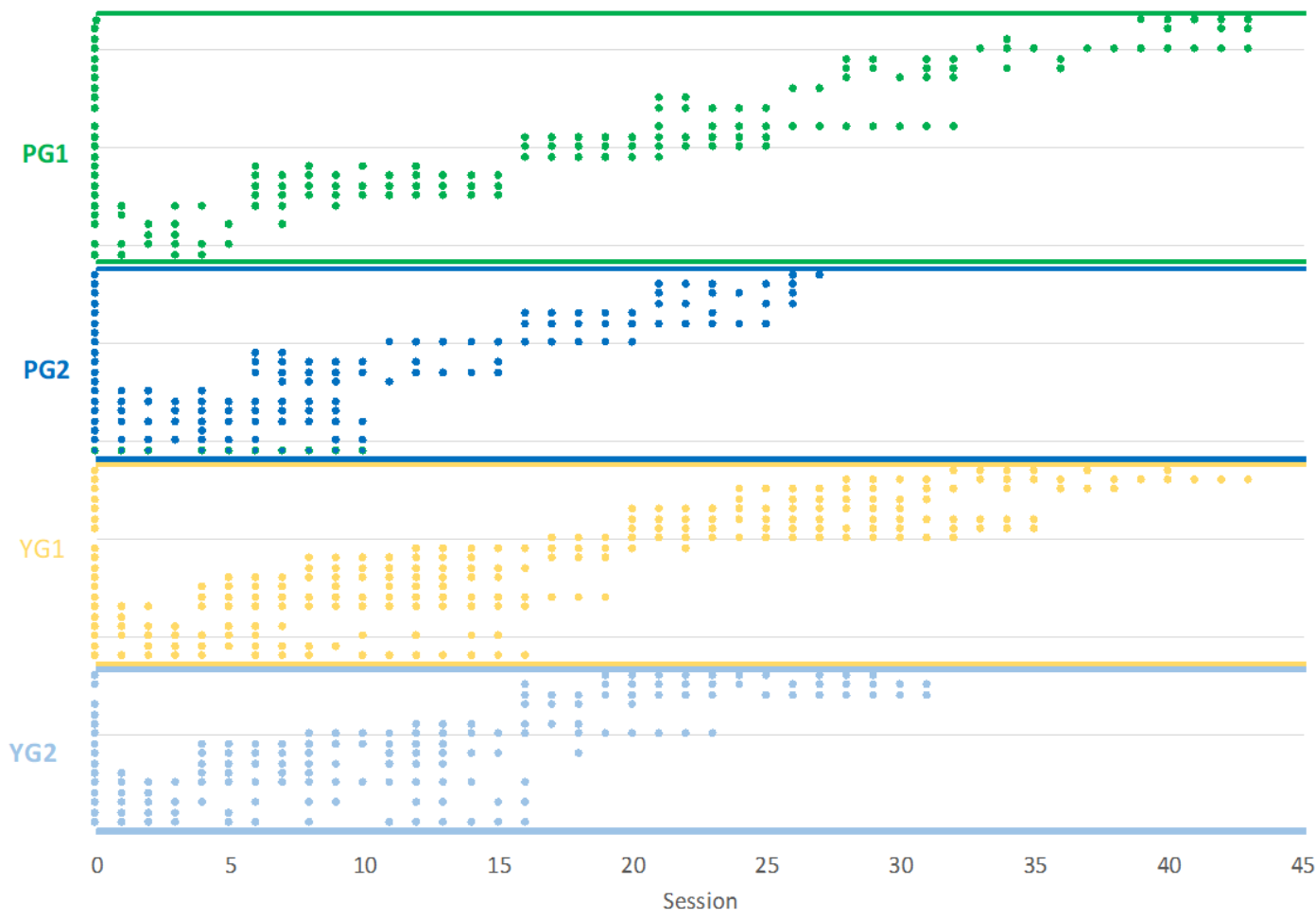

Figure 3. Participation of the trainees at the different sessions. 


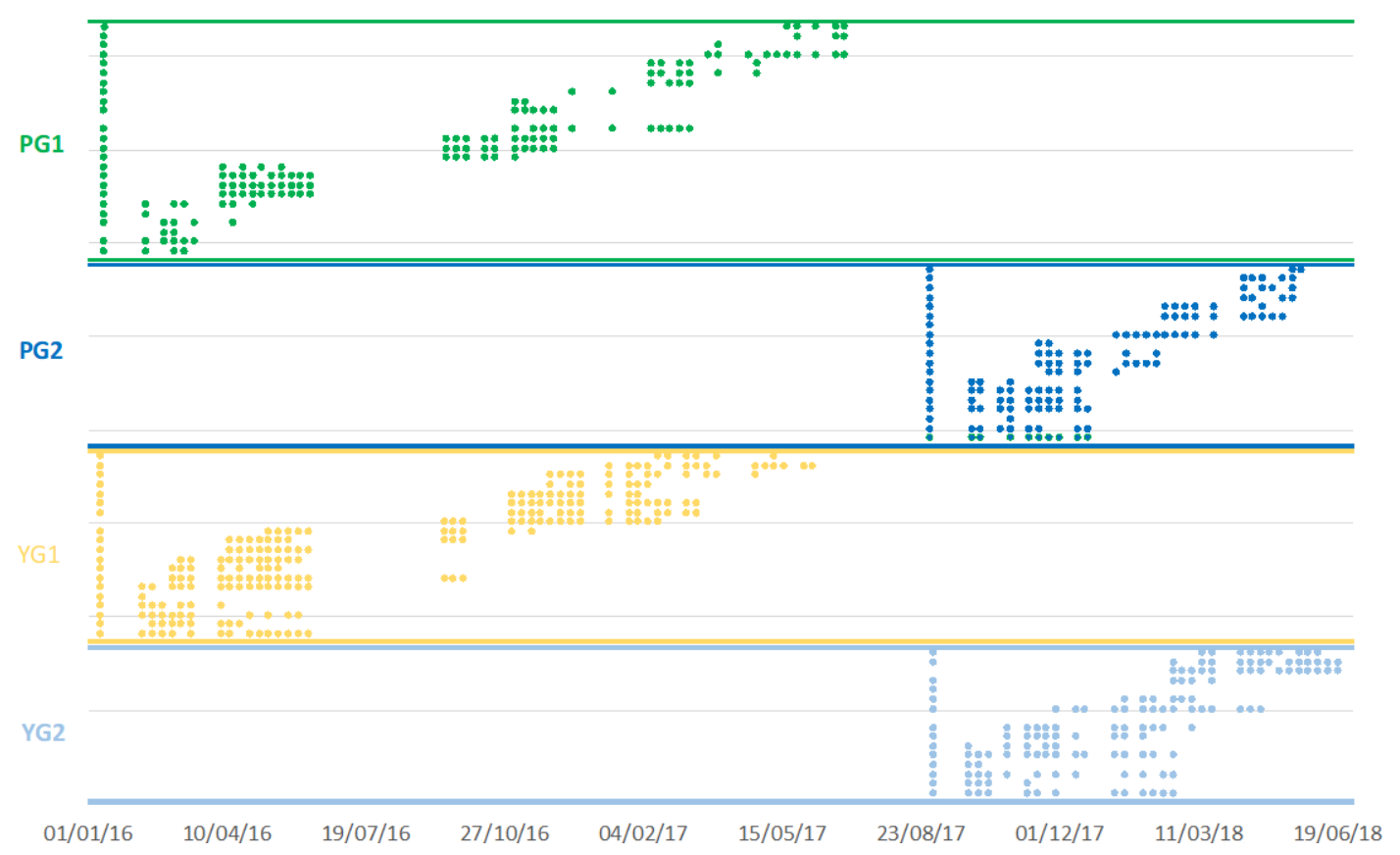

Figure 4. Evolution of the attendance to the training versus time.

\subsection{Data Analysis}

The most frequently chosen picture in each pair during the "zero" test (the questionnaire administered before the first meeting) is indicated as picture $\mathrm{A}\left(\mathrm{A}_{i}, i=\right.$ $1.50)$, while the other picture is designed as $\mathrm{B}\left(\mathrm{B}_{i}, i=1.50\right)$. We have computed frequency tables for each pair of images and each session for the four groups. Because the present work is focused on the influence of the group unconscious on the measured effects-i.e., the answers to the questionnaire-we consider only the proportion of the participants choosing picture A or B for each of the 50 questions, irrespectively of how the individual participant's choice evolved.

Given the very uneven participation, we decided not to correct for data that were completely missing. Some of the sheets were incorrectly marked, and those we corrected with an LOCF (Last Observation Carry Forward, (Hamer \& Simpson, 2009)) algorithm, using the same answer from the previous session. If this was not possible, the session before was used, and so on. There was no instance of invalid questionnaires for both the zero and first sessions. The total number of corrections is reported in Table 2.

\section{Results}

\subsection{Evolution of the Most Chosen Picture}

We report the evolution of the A's (most chosen picture in questionnaire zero) for the four groups during the training. We have compared the percentage of the "A" picture choice for the 50 questions in the successive sessions. For this, we have performed a Friedmann test on the whole set of the <number of sessions $>$ $\times 50$ data, and then, if significant-which has always been the case-we have performed a post-hoc Conover test (Conover, 1999; Conover \& Iman, 1979) between 
Table 2. Corrections to the data with LOCF.

\begin{tabular}{cccc}
\hline Group & Total valid answers & Answers corrected with LOCF & $\%$ \\
\hline PG1 & 8148 & 101 & $1.2 \%$ \\
PG2 & 6406 & 95 & $1.5 \%$ \\
YG1 & 11,131 & 69 & $0.6 \%$ \\
YG2 & 7037 & 63 & 0.9 \\
\hline
\end{tabular}

successive sessions. All the data analysis has been done with the $\mathrm{R}$ software ( $\mathrm{R}$ Core Team, 2020).

We show the statistically significant difference in the choice of A's between sessions, including the initial questionnaire (see Figure 5 and Table 3).

\subsection{Evolution of the Transitions between Questionnaires}

We consider now how the choice of A and B has evolved comparing the changes of choice of the participants. For each couple of successive sessions, we count the number of participants whose choice has changed from A to B and the number of those whose choice has changed from $\mathrm{B}$ to $\mathrm{A}$. We indicate this transition with the number of the second session, i.e., we indicate with $n$ the transition $(A \rightarrow B$ o0 $\mathrm{B} \rightarrow \mathrm{A}$ ) between session $n-1$ and session $n$. To avoid repetitions, we will refer to this explanation as the notation explained in the section "Evolution of the transition between questionnaires."

We first consider the number of changes from $A \rightarrow B$. The statistical differences we found for the four groups are reported in Table 4 and Figure 6.

Now we consider the same statistics but for the transitions $B \rightarrow A$ for the four groups. The results are shown in Figure 7 and Table 5.

We now investigate whether the individual changes of choice $A \rightarrow B$ and $B \rightarrow A$ are simple statistical fluctuations in people's preference or whether there is a statistically significant difference. This may indicate that the change of choice of the group taken as an ensemble is not casual and that there is a significant movement either toward the initial choice or away from it. As before, we indicate with $n$ the change from $\mathrm{A} \rightarrow \mathrm{B}$ or $\mathrm{B} \rightarrow \mathrm{A}$ between session $n-1$ and session $n$. We will call this the non-casuality plot.

We found statistically significant differences, considering the four groups, with the notation explained in the session "Evolution of the transitions between questionnaires," as shown in Figure 8 and Table 6.

\subsection{Evolution of "Flux" and "Focus"}

We can combine the transitions $\mathrm{A} \rightarrow \mathrm{B}$ and $\mathrm{B} \rightarrow \mathrm{A}$ into two derived quantities that help to interpret the evolution of the groups' choice of pictures. We call the quantity $A \rightarrow B+B \rightarrow A$ the "flux" as it expresses the total "activity" of the group in changing the initial choice of picture. We introduce a second quantity, $B \rightarrow A-A \rightarrow B$, that we call "focus" as it indicates the tendency to confirm, if greater than 0 , or move away from, if smaller than 0 , the initial choice of A. 
A Parents G1

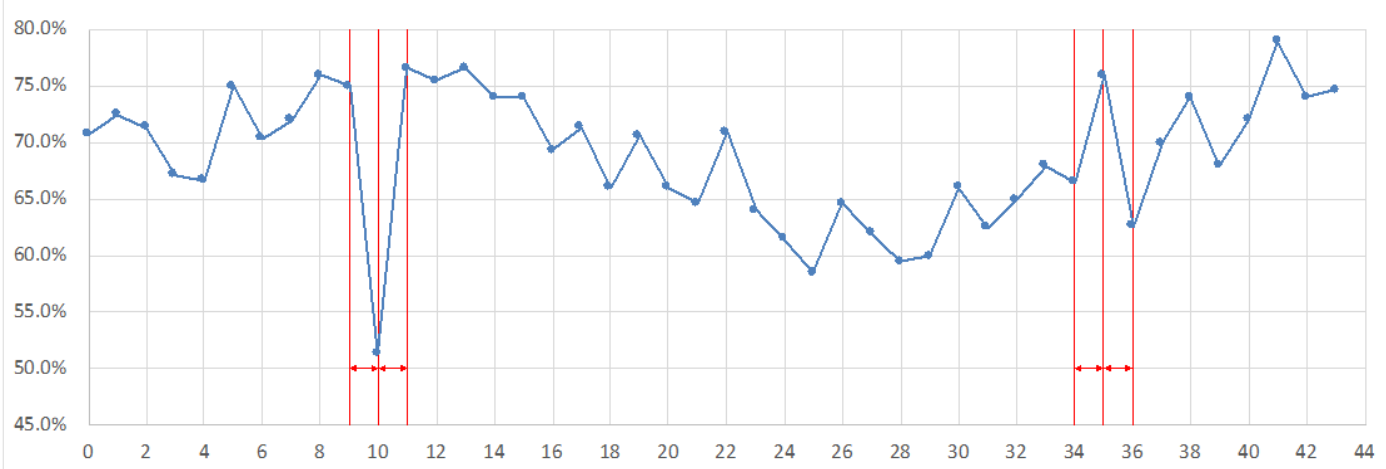

A Parents G2

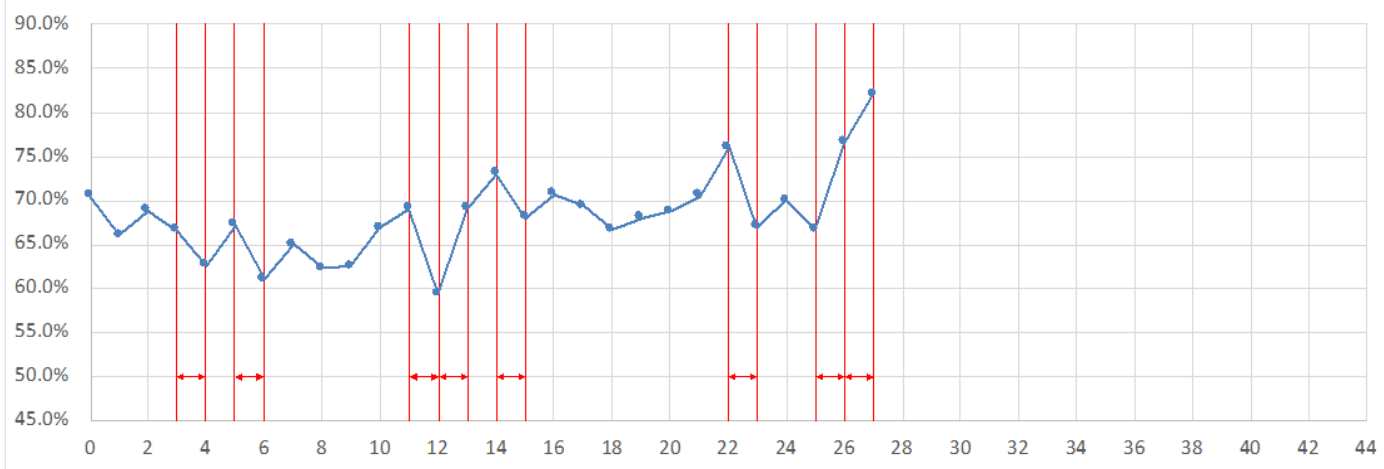

A Young G1

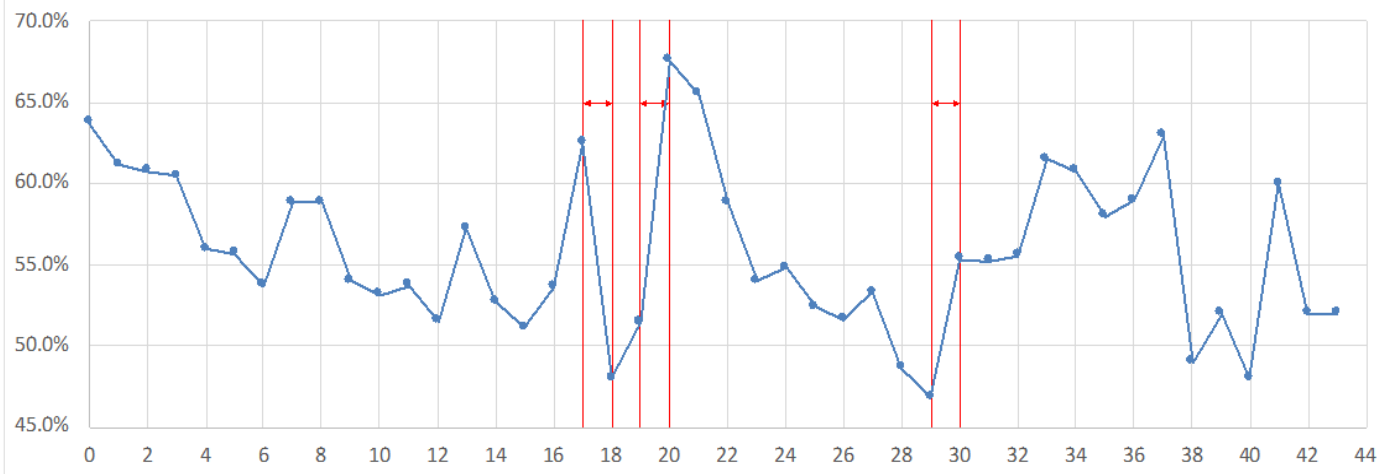

A Young G2

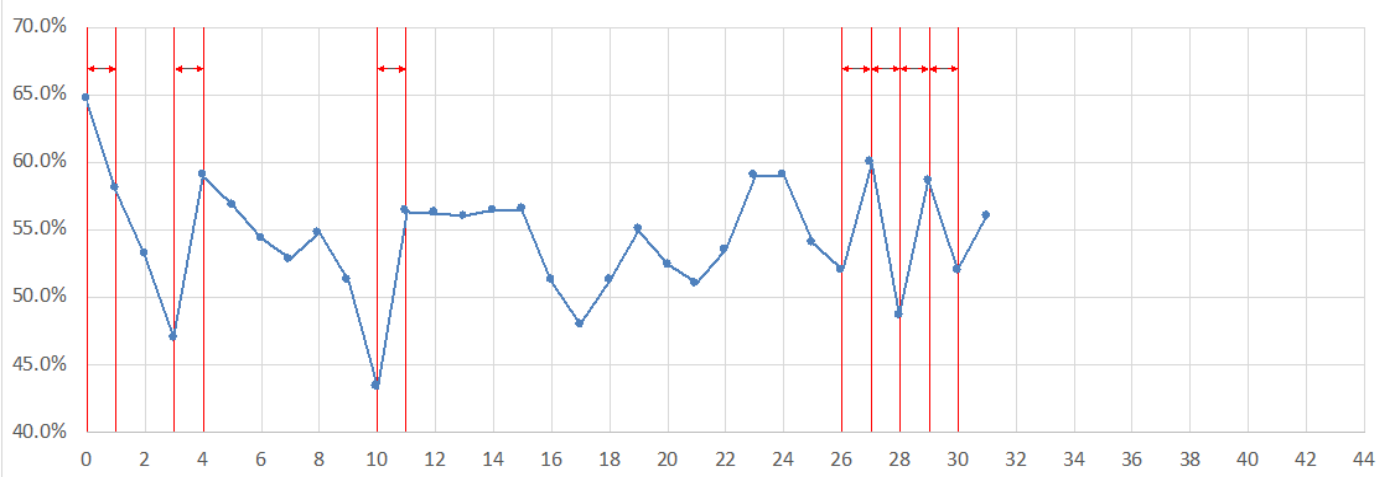

Figure 5. Evolution of the choice of A for the four groups during the training versus the session number. The arrows mark the couple of sessions for which there is a statistically significant difference $(p<0.05)$ with the Conover post-hoc test. 

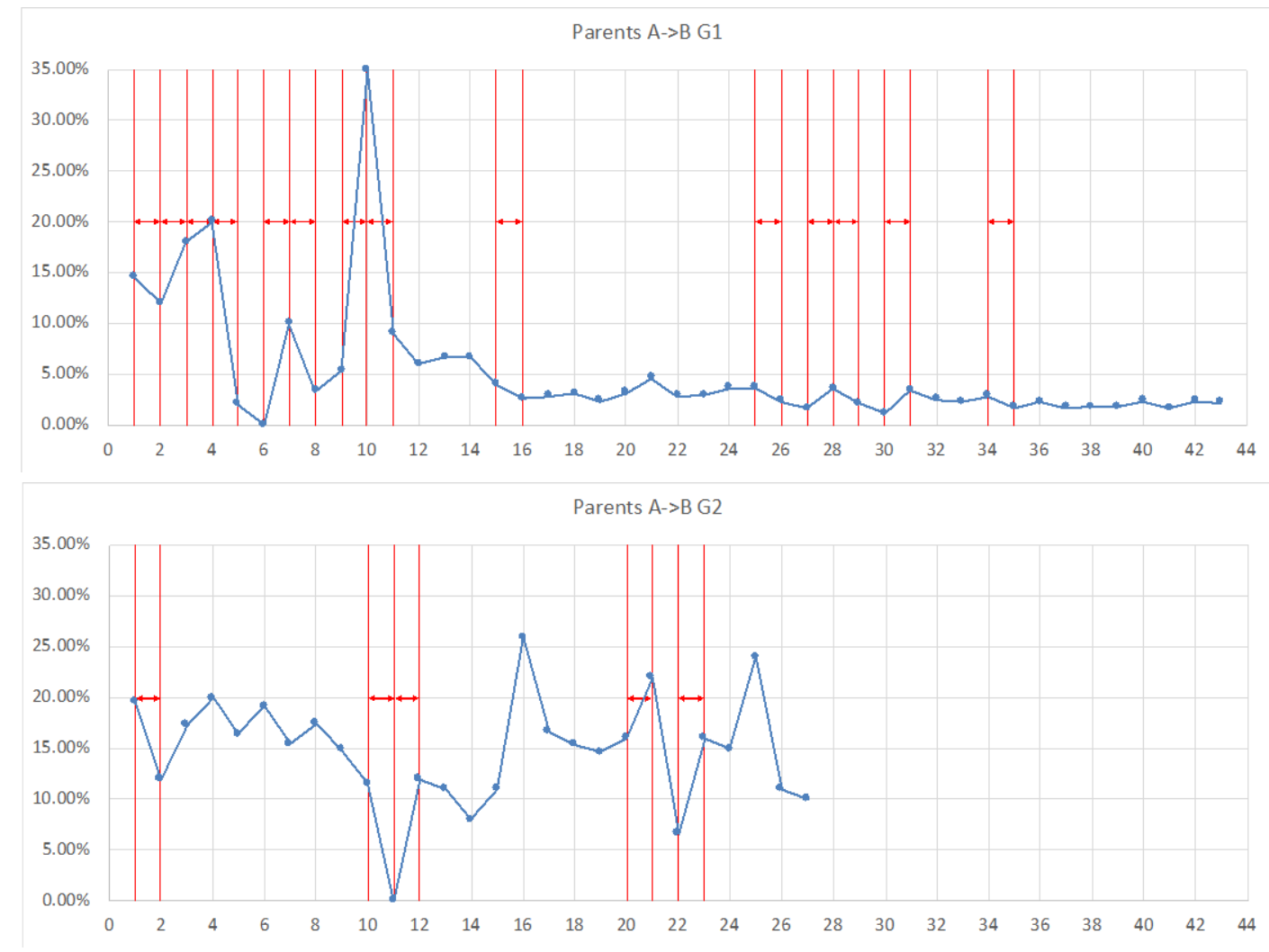

Young $A->B$ G1
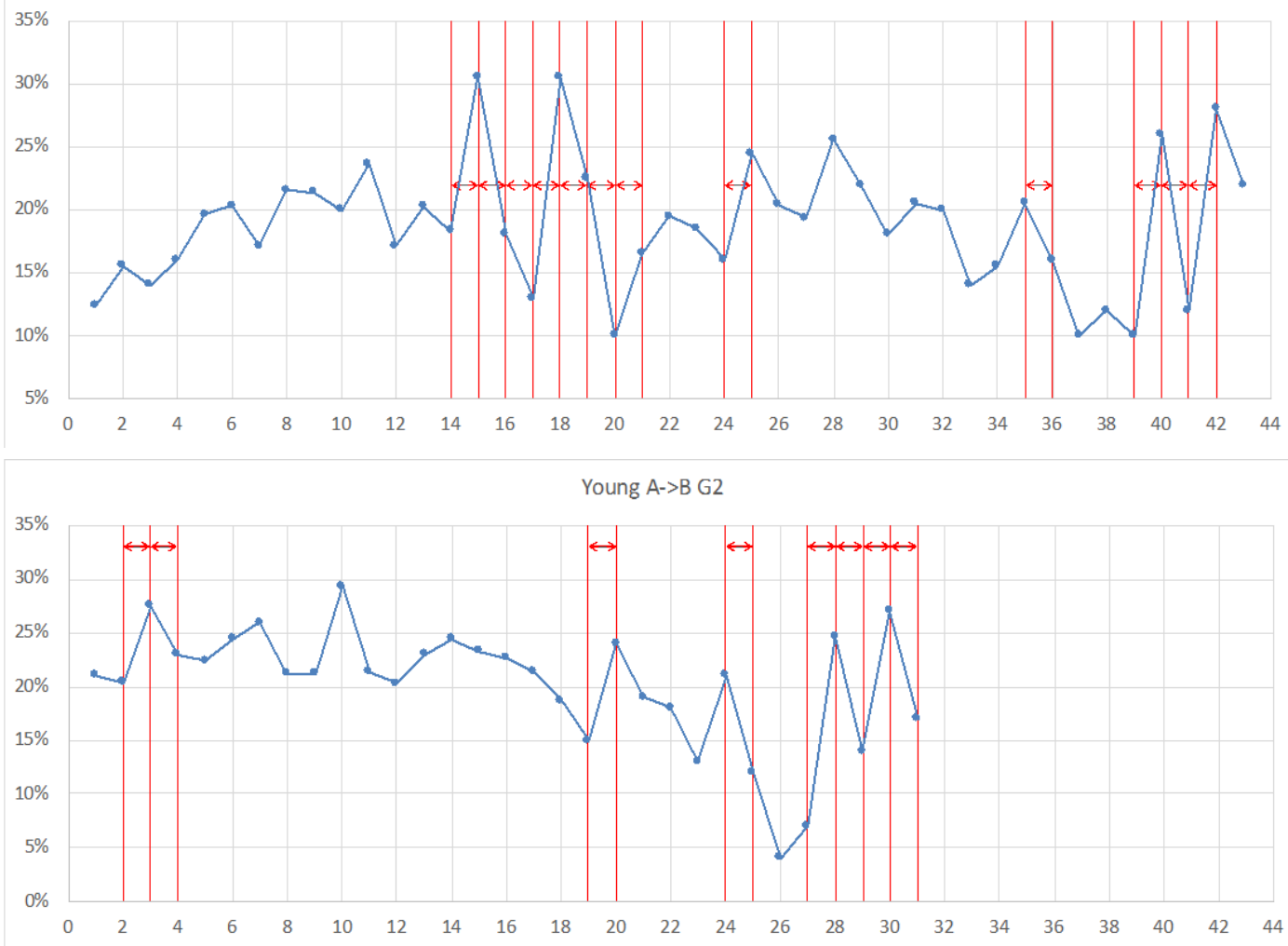

Figure 6. Evolution of the change in choice $A \rightarrow B$ for the four groups during the training versus the session number. The arrows mark the couple of sessions for which there is a statistically significant difference $(p<$ 0.05) with the Conover post-hoc test. 

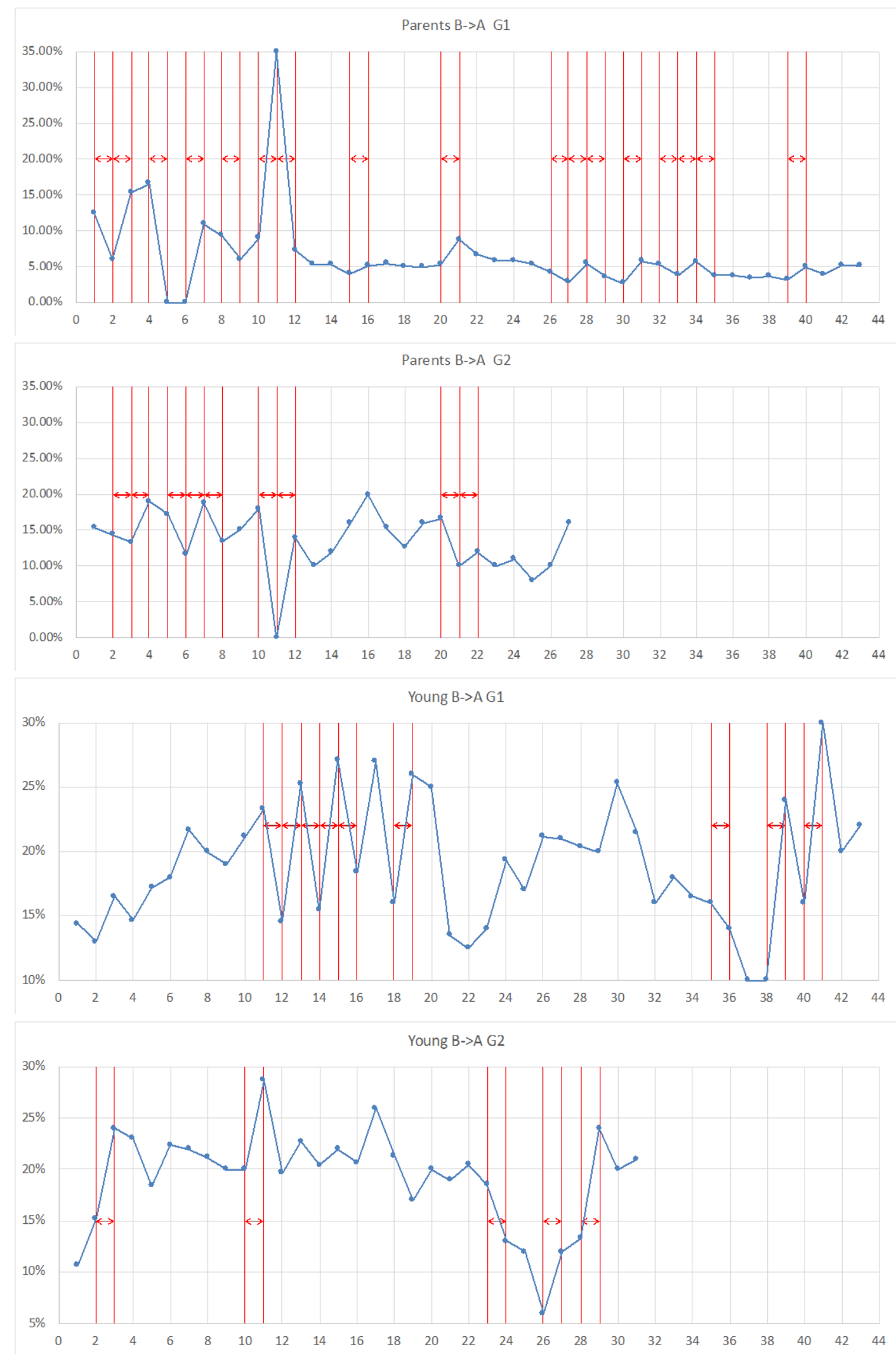

Figure 7. Evolution of the change in choice $B \rightarrow A$ for the four groups during the training versus the session number. The arrows mark the couple of sessions for which there is a statistically significant difference $(p<$ 0.05) with the Conover post-hoc test. 

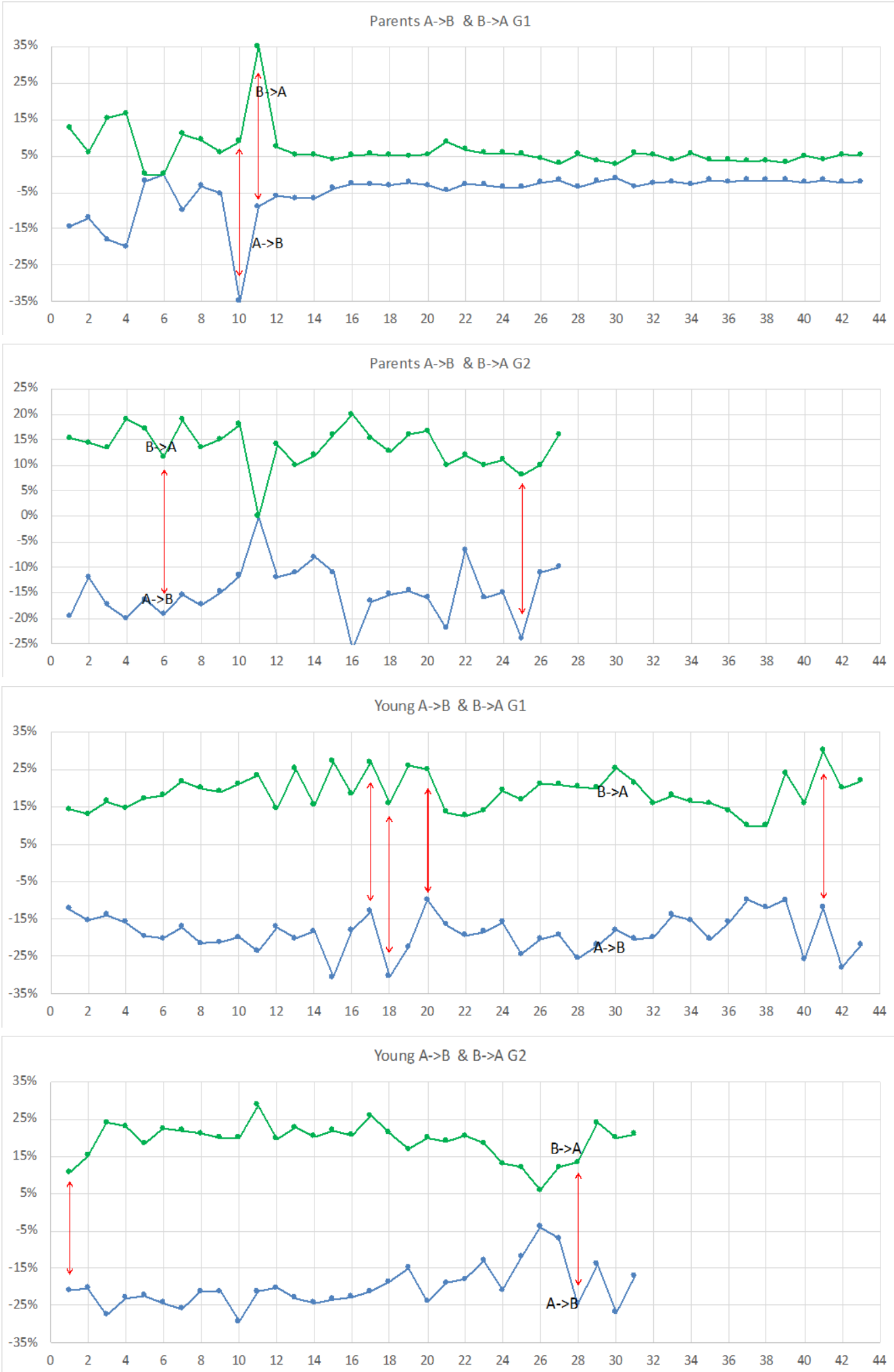

Figure 8. Comparison of the percentage of $\mathrm{A} \rightarrow \mathrm{B}$ and $\mathrm{B} \rightarrow \mathrm{A}$ changes for each group as calculated with the Conover test following a Friedmann test on the totality of the percentages. We report on the graphs the average of the percentages of changes of each type for each session. A red arrow indicates the transitions for which there is a significant difference $(p<0.05)$ between the $A \rightarrow B$ and $B \rightarrow A$ percentages for the 50 questions. For clarity, we report the negative of the average of the $A \rightarrow B$ percentages. 
Table 3. Comparison of the choices of the "A picture" between successive sessions with the Conover post-hoc test. Two sessions are statistically different when $p<0.05$, and the corresponding cell is red in the table.

\begin{tabular}{cccccccccc}
\hline Comparison & PG1 & PG2 & YG1 & YG2 & Comparison & PG1 & PG2 & YG1 & YG2 \\
\hline A00 - A01 & 0.54 & 0.37 & 0.43 & 0.02 & A22 - A23 & 0.12 & 0.03 & 0.25 & 0.09 \\
A01 - A02 & 0.81 & 0.26 & 0.53 & 0.15 & A23 - A24 & 0.59 & 0.70 & 0.97 & 0.87 \\
A02 - A03 & 0.51 & 0.75 & 0.98 & 0.06 & A24 - A25 & 0.53 & 0.20 & 0.55 & 0.31 \\
A03 - A04 & 0.73 & 0.04 & 0.35 & 0.00 & A25 - A26 & 0.17 & 0.00 & 0.93 & 0.44 \\
A04 - A05 & 0.05 & 0.10 & 0.91 & 0.98 & A26 - A27 & 0.85 & 0.03 & 0.68 & 0.02 \\
A05 - A06 & 0.23 & 0.05 & 0.61 & 0.13 & A27 - A28 & 0.20 & & 0.36 & 0.01 \\
A06 - A07 & 0.47 & 0.37 & 0.22 & 0.89 & A28 - A29 & 0.53 & & 0.49 & 0.02 \\
A07 - A08 & 0.47 & 0.57 & 0.93 & 0.57 & A29 - A30 & 0.18 & & 0.05 & 0.04 \\
A08 - A09 & 0.74 & 0.90 & 0.18 & 0.25 & A30 - A31 & 0.33 & & 0.69 & 0.38 \\
A09 - A10 & 0.00 & 0.15 & 0.90 & 0.08 & A31 - A32 & 0.69 & & 0.78 & \\
A10 - A11 & 0.00 & 0.17 & 0.67 & 0.00 & A32 - A33 & 0.32 & & 0.39 & \\
A11 - A12 & 1.00 & 0.00 & 0.45 & 0.88 & A33 - A34 & 0.60 & & 0.99 & \\
A12 - A13 & 0.81 & 0.00 & 0.19 & 1.00 & A34 - A35 & 0.03 & & 0.51 & \\
A13 - A14 & 0.49 & 0.42 & 0.38 & 0.92 & A35 - A36 & 0.00 & & 0.92 & \\
A14 - A15 & 0.93 & 0.02 & 0.67 & 0.97 & A36 - A37 & 0.07 & & 0.46 & \\
A15 - A16 & 0.27 & 0.14 & 0.44 & 0.17 & A37 - A38 & 0.40 & & 0.01 & \\
A16 - A17 & 0.56 & 0.51 & 0.06 & 0.53 & A38 - A39 & 0.25 & & 0.19 & \\
A17 - A18 & 0.23 & 0.92 & 0.00 & 0.70 & A39 - A40 & 0.50 & & 0.12 & \\
A18 - A19 & 0.42 & 0.97 & 0.52 & 0.48 & A40 - A41 & 0.10 & & 0.00 & \\
A19 - A20 & 0.58 & 0.80 & 0.00 & 0.76 & A41 - A42 & 0.16 & & 0.15 & \\
A20 - A21 & 0.45 & 0.36 & 0.44 & 0.74 & A42 - A43 & 0.79 & & 0.95 & \\
A21 - A22 & 0.10 & 0.15 & 0.16 & 0.67 & & & & & \\
\hline
\end{tabular}

Table 4. Comparison of the change of choices $A \rightarrow B$ between successive sessions with the Conover post-hoc test. Two changes of choices are statistically different when $p<0.05$, and the corresponding cell is red in the table.

\begin{tabular}{llllllllll}
\hline Change $A \rightarrow B$ & PG1 & PG2 & YG1 & YG2 & Change $A \rightarrow B$ & PG1 & PG2 & YG1 & YG2 \\
\hline $00 \rightarrow 01-01 \rightarrow 02$ & 0.00 & 0.00 & 0.24 & 0.69 & $21 \rightarrow 22-22 \rightarrow 23$ & 0.79 & 0.04 & 0.71 & 0.12 \\
$01 \rightarrow 02-02 \rightarrow 03$ & 0.00 & 0.24 & 0.40 & 0.04 & $22 \rightarrow 23-23 \rightarrow 24$ & 0.43 & 0.92 & 0.39 & 0.18 \\
$02 \rightarrow 03-03 \rightarrow 04$ & 0.01 & 0.09 & 0.46 & 0.01 & $23 \rightarrow 24-24 \rightarrow 25$ & 0.99 & 0.54 & 0.01 & 0.00 \\
$03 \rightarrow 04-04 \rightarrow 05$ & 0.00 & 0.91 & 0.27 & 0.16 & $24 \rightarrow 25-25 \rightarrow 26$ & 0.04 & 0.43 & 0.43 & 0.14 \\
$04 \rightarrow 05-05 \rightarrow 06$ & 0.66 & 0.64 & 0.31 & 0.63 & $25 \rightarrow 26-26 \rightarrow 27$ & 0.25 & 0.08 & 0.90 & 0.15 \\
$05 \rightarrow 06-06 \rightarrow 07$ & 0.00 & 0.07 & 0.26 & 0.86 & $26 \rightarrow 27-27 \rightarrow 28$ & 0.01 & & 0.42 & 0.00 \\
$06 \rightarrow 07-07 \rightarrow 08$ & 0.00 & 0.13 & 0.26 & 0.23 & $27 \rightarrow 28-28 \rightarrow 29$ & 0.03 & & 0.67 & 0.01 \\
$07 \rightarrow 08-08 \rightarrow 09$ & 0.15 & 0.16 & 0.72 & 0.83 & $28 \rightarrow 29-29 \rightarrow 30$ & 0.14 & & 0.19 & 0.01 \\
$08 \rightarrow 09-09 \rightarrow 10$ & 0.00 & 0.14 & 0.77 & 0.18 & $29 \rightarrow 30-30 \rightarrow 31$ & 0.00 & & 0.46 & 0.02 \\
$09 \rightarrow 10-10 \rightarrow 11$ & 0.00 & 0.00 & 0.32 & 0.07 & $30 \rightarrow 31-31 \rightarrow 32$ & 0.13 & & 0.21 & \\
$10 \rightarrow 11-11 \rightarrow 12$ & 0.61 & 0.02 & 0.09 & 0.53 & $31 \rightarrow 32-32 \rightarrow 33$ & 0.90 & & 0.16 & \\
$11 \rightarrow 12-12 \rightarrow 13$ & 1.00 & 0.34 & 0.27 & 0.38 & $32 \rightarrow 33-33 \rightarrow 34$ & 0.45 & & 0.29 & \\
$12 \rightarrow 13-13 \rightarrow 14$ & 0.72 & 0.42 & 0.52 & 0.99 & $33 \rightarrow 34-34 \rightarrow 35$ & 0.04 & & 0.17 & \\
$13 \rightarrow 14-14 \rightarrow 15$ & 0.21 & 0.27 & 0.00 & 0.38 & $34 \rightarrow 35-35 \rightarrow 36$ & 0.30 & & 0.00 & \\
$14 \rightarrow 15-15 \rightarrow 16$ & 0.00 & 0.18 & 0.00 & 0.83 & $35 \rightarrow 36-36 \rightarrow 37$ & 0.30 & & 0.36 & \\
$15 \rightarrow 16-16 \rightarrow 17$ & 0.85 & 0.10 & 0.01 & 0.53 & $36 \rightarrow 37-37 \rightarrow 38$ & 0.86 & & 0.78 & \\
$16 \rightarrow 17-17 \rightarrow 18$ & 0.70 & 0.34 & 0.00 & 0.30 & $37 \rightarrow 38-38 \rightarrow 39$ & 0.94 & & 0.74 & \\
$17 \rightarrow 18-18 \rightarrow 19$ & 0.19 & 0.84 & 0.04 & 0.16 & $38 \rightarrow 39-39 \rightarrow 40$ & 0.37 & & 0.01 & \\
$18 \rightarrow 19-19 \rightarrow 20$ & 0.10 & 0.55 & 0.00 & 0.00 & $39 \rightarrow 40-40 \rightarrow 41$ & 0.23 & & 0.02 & \\
$19 \rightarrow 20-20 \rightarrow 21$ & 0.25 & 0.04 & 0.00 & 0.16 & $40 \rightarrow 41-41 \rightarrow 42$ & 0.19 & & 0.01 & \\
$20 \rightarrow 21-21 \rightarrow 22$ & 0.08 & 0.17 & 0.56 & 0.81 & $41 \rightarrow 42-42 \rightarrow 43$ & 0.71 & & 0.34 & \\
\hline
\end{tabular}


Table 5. Comparison of the change of choices $B \rightarrow A$ between successive sessions with the Conover post-hoc test. Two changes of choices are statistically different when $p<0.05$, and the corresponding cell is red in the table.

\begin{tabular}{llllllllll}
\hline Change B $\rightarrow$ A & PG1 & PG2 & YG1 & YG2 & Change B $\rightarrow$ A & PG1 & PG2 & YG1 & YG2 \\
\hline $00 \rightarrow 01-01 \rightarrow 02$ & 0.00 & 0.59 & 0.74 & 0.18 & $21 \rightarrow 22-22 \rightarrow 23$ & 0.25 & 0.09 & 0.57 & 0.63 \\
$01 \rightarrow 02-02 \rightarrow 03$ & 0.00 & 0.02 & 0.27 & 0.01 & $22 \rightarrow 23-23 \rightarrow 24$ & 0.96 & 0.62 & 0.27 & 0.01 \\
$02 \rightarrow 03-03 \rightarrow 04$ & 0.07 & 0.01 & 0.48 & 0.13 & $23 \rightarrow 24-24 \rightarrow 25$ & 0.66 & 0.05 & 0.83 & 0.14 \\
$03 \rightarrow 04-04 \rightarrow 05$ & 0.00 & 0.91 & 0.50 & 0.79 & $24 \rightarrow 25-25 \rightarrow 26$ & 0.12 & 0.07 & 0.21 & 0.31 \\
$04 \rightarrow 05-05 \rightarrow 06$ & 1.00 & 0.05 & 0.37 & 0.20 & $25 \rightarrow 26-26 \rightarrow 27$ & 0.05 & 0.76 & 0.94 & 0.01 \\
$05 \rightarrow 06-06 \rightarrow 07$ & 0.00 & 0.00 & 0.27 & 0.93 & $26 \rightarrow 27-27 \rightarrow 28$ & 0.00 & & 0.70 & 0.33 \\
$06 \rightarrow 07-07 \rightarrow 08$ & 0.11 & 0.01 & 0.34 & 0.60 & $27 \rightarrow 28-28 \rightarrow 29$ & 0.01 & & 0.83 & 0.02 \\
$07 \rightarrow 08-08 \rightarrow 09$ & 0.02 & 0.28 & 0.89 & 0.78 & $28 \rightarrow 29-29 \rightarrow 30$ & 0.19 & & 0.18 & 0.31 \\
$08 \rightarrow 09-09 \rightarrow 10$ & 0.33 & 0.80 & 0.68 & 0.41 & $29 \rightarrow 30-30 \rightarrow 31$ & 0.00 & & 0.08 & 0.71 \\
$09 \rightarrow 10-10 \rightarrow 11$ & 0.00 & 0.00 & 0.58 & 0.02 & $30 \rightarrow 31-31 \rightarrow 32$ & 0.53 & & 0.09 & \\
$10 \rightarrow 11-11 \rightarrow 12$ & 0.00 & 0.01 & 0.01 & 0.12 & $31 \rightarrow 32-32 \rightarrow 33$ & 0.03 & & 0.67 & \\
$11 \rightarrow 12-12 \rightarrow 13$ & 0.12 & 0.69 & 0.00 & 0.12 & $32 \rightarrow 33-33 \rightarrow 34$ & 0.01 & & 0.91 & \\
$12 \rightarrow 13-13 \rightarrow 14$ & 0.57 & 0.75 & 0.02 & 0.24 & $33 \rightarrow 34-34 \rightarrow 35$ & 0.00 & & 0.83 & \\
$13 \rightarrow 14-14 \rightarrow 15$ & 0.38 & 0.13 & 0.00 & 0.48 & $34 \rightarrow 35-35 \rightarrow 36$ & 0.93 & & 0.00 & \\
$14 \rightarrow 15-15 \rightarrow 16$ & 0.00 & 0.28 & 0.00 & 0.95 & $35 \rightarrow 36-36 \rightarrow 37$ & 0.65 & & 0.56 & \\
$15 \rightarrow 16-16 \rightarrow 17$ & 0.59 & 0.11 & 0.36 & 0.31 & $36 \rightarrow 37-37 \rightarrow 38$ & 0.76 & & 0.95 & \\
$16 \rightarrow 17-17 \rightarrow 18$ & 0.50 & 0.79 & 0.07 & 0.11 & $37 \rightarrow 38-38 \rightarrow 39$ & 0.72 & & 0.03 & \\
$17 \rightarrow 18-18 \rightarrow 19$ & 0.93 & 0.26 & 0.00 & 0.41 & $38 \rightarrow 39-39 \rightarrow 40$ & 0.02 & & 0.20 & \\
$18 \rightarrow 19-19 \rightarrow 20$ & 0.55 & 0.51 & 0.06 & 0.06 & $39 \rightarrow 40-40 \rightarrow 41$ & 0.09 & & 0.03 & \\
$19 \rightarrow 20-20 \rightarrow 21$ & 0.02 & 0.00 & 0.09 & 0.72 & $40 \rightarrow 41-41 \rightarrow 42$ & 0.05 & & 0.11 & \\
$20 \rightarrow 21-21 \rightarrow 22$ & 0.47 & 0.00 & 0.75 & 0.88 & $41 \rightarrow 42-42 \rightarrow 43$ & 0.96 & & 0.67 & \\
\hline & & & & & & & & & \\
& & & & &
\end{tabular}

Table 6. Non-Casuality, statistically significant differences $(p<0.05)$ in the percentage of transitions of choice $\mathrm{B} \rightarrow \mathrm{A}$ vs. $\mathrm{A} \rightarrow \mathrm{B}$ for the same session. The cells in red represent statistically significant $(p<0.05)$ differences between the percentages of change in the picture choice $\mathrm{A} \rightarrow \mathrm{B}$ and $\mathrm{B} \rightarrow \mathrm{A}$, indicating a deviation from a purely statistical fluctuation of the choices.

\begin{tabular}{|c|c|c|c|c|c|c|c|c|c|}
\hline & PG1 & PG2 & YG1 & YG2 & & PG1 & PG2 & YG1 & YG2 \\
\hline S00-01 & 0.90 & 0.51 & 0.64 & 0.01 & S22-23 & 0.29 & 0.15 & 0.45 & 0.19 \\
\hline S01-02 & 0.50 & 0.22 & 0.59 & 0.22 & S23-24 & 0.51 & 0.23 & 0.60 & 0.12 \\
\hline S02-03 & 0.87 & 0.25 & 0.41 & 0.41 & S24-25 & 0.64 & 0.03 & 0.15 & 0.99 \\
\hline S03-04 & 0.33 & 0.48 & 0.81 & 0.93 & S25-26 & 0.37 & 0.79 & 0.94 & 0.79 \\
\hline S04-05 & 0.83 & 0.54 & 0.54 & 0.33 & S26-27 & 0.36 & 0.42 & 0.98 & 0.29 \\
\hline S05-06 & 1.00 & 0.02 & 0.55 & 0.70 & S27-28 & 0.41 & & 0.39 & 0.04 \\
\hline S06-07 & 0.76 & 0.13 & 0.34 & 0.57 & S28-29 & 0.36 & & 0.72 & 0.16 \\
\hline S07-08 & 0.12 & 0.17 & 0.66 & 0.96 & S29-30 & 0.31 & & 0.13 & 0.30 \\
\hline S08-09 & 0.79 & 0.67 & 0.91 & 0.88 & $\mathrm{~S} 30-31$ & 0.36 & & 0.84 & 0.42 \\
\hline S09-10 & 0.00 & 0.20 & 0.68 & 0.10 & S31-32 & 0.15 & & 0.50 & \\
\hline S10-11 & 0.00 & 1.00 & 0.88 & 0.19 & S32-33 & 0.64 & & 0.49 & \\
\hline $\mathrm{S} 11-12$ & 0.88 & 0.78 & 0.55 & 0.76 & S33-34 & 0.25 & & 0.88 & \\
\hline S12-13 & 0.63 & 0.97 & 0.48 & 0.90 & S34-35 & 0.21 & & 0.37 & \\
\hline S13-14 & 0.64 & 0.45 & 0.61 & 0.50 & S35-36 & 0.56 & & 0.83 & \\
\hline S14-15 & 0.91 & 0.31 & 0.71 & 0.63 & S36-37 & 0.33 & & 0.99 & \\
\hline S15-16 & 0.33 & 0.45 & 0.89 & 0.69 & S37-38 & 0.27 & & 0.83 & \\
\hline S16-17 & 0.22 & 0.42 & 0.01 & 0.44 & S38-39 & 0.28 & & 0.11 & \\
\hline S17-18 & 0.45 & 0.76 & 0.00 & 0.74 & S39-40 & 0.15 & & 0.25 & \\
\hline S18-19 & 0.16 & 0.74 & 0.51 & 0.46 & S40-41 & 0.14 & & 0.04 & \\
\hline S19-20 & 0.55 & 0.61 & 0.01 & 0.60 & S41-42 & 0.14 & & 0.35 & \\
\hline S20-21 & 0.30 & 0.09 & 0.39 & 0.72 & $\mathrm{~S} 42-43$ & 0.10 & & 0.97 & \\
\hline S21-22 & 0.18 & 0.19 & 0.15 & 0.63 & & & & & \\
\hline
\end{tabular}


As in the previous section, we consider at first a Friedmann test of the whole $50 \mathrm{x}<$ number of sessions $>$ matrix for the focus for the four groups. All these tests indicate a significant deviation from purely random fluctuations. Following this, we compare each session with the following one using the Conover posthoc test for each group. The results can be seen in Figure 9 and Table 7.
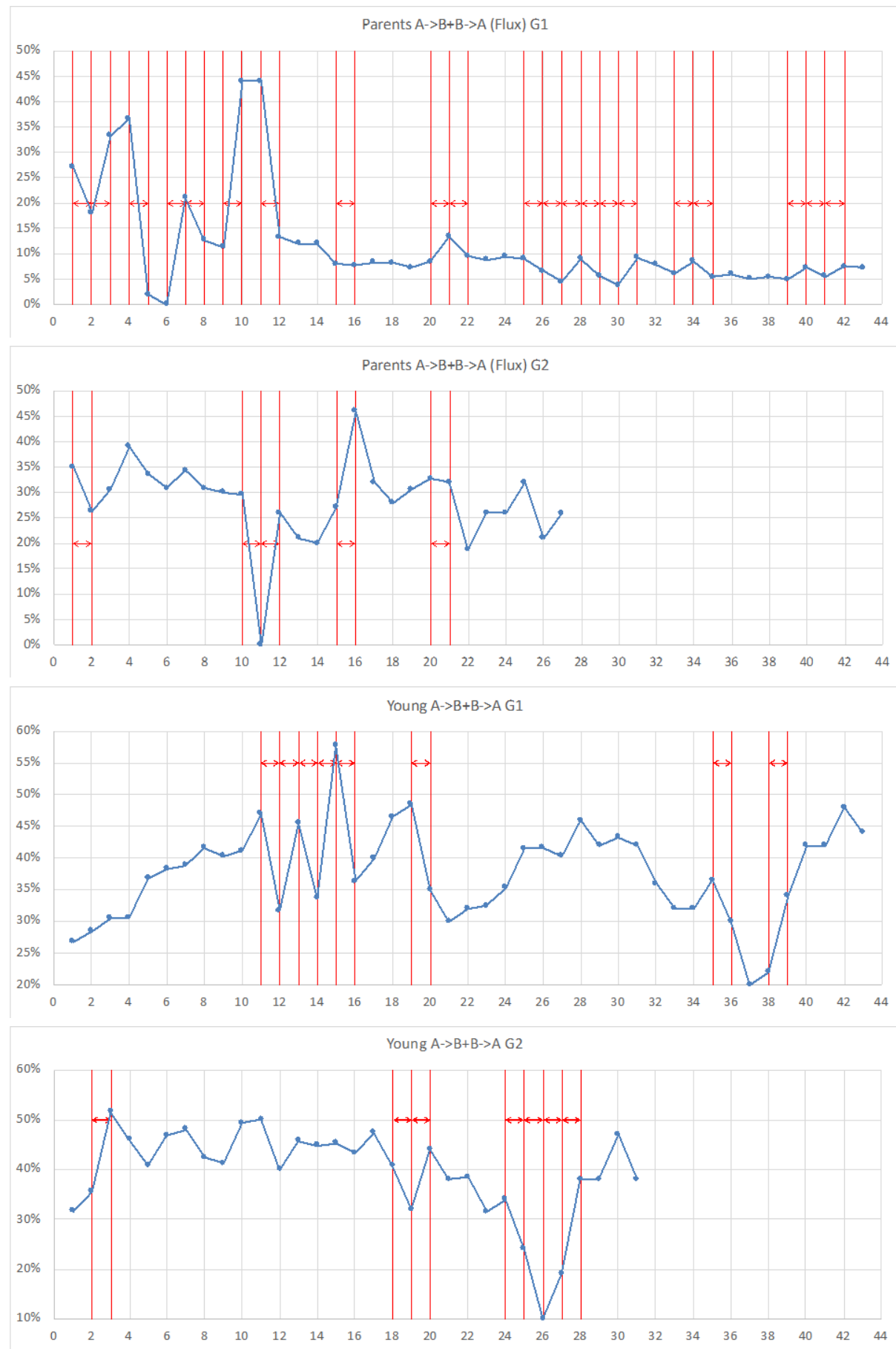

Figure 9. Comparison of the percentage of $A \rightarrow B+B \rightarrow A$ changes between successive sessions for each group as calculated with the Conover test following a Friedmann test on the totality of the percentages. 
Table 7. Comparison of the flux of choices $A \rightarrow B+B \rightarrow A$ between successive sessions with the Conover post-hoc test. Two changes of choices are statistically different when $p<$ 0.05 , and the corresponding cell is red in the table.

\begin{tabular}{cccccccccc}
\hline $\mathrm{A} \rightarrow \mathrm{B}+\mathrm{B} \rightarrow \mathrm{A}$ & PG1 & PG2 & YG1 & YG2 & $\mathrm{A} \rightarrow \mathrm{B}+\mathrm{B} \rightarrow \mathrm{A}$ & PG1 & PG2 & YG1 & YG2 \\
\hline $00 \rightarrow 01-01 \rightarrow 02$ & 0.00 & 0.01 & 0.49 & 0.30 & $21 \rightarrow 22-22 \rightarrow 23$ & 0.45 & 0.11 & 0.96 & 0.10 \\
$01 \rightarrow 02-02 \rightarrow 03$ & 0.00 & 0.33 & 0.77 & 0.00 & $22 \rightarrow 23-23 \rightarrow 24$ & 0.55 & 0.97 & 0.62 & 0.51 \\
$02 \rightarrow 03-03 \rightarrow 04$ & 0.29 & 0.07 & 0.87 & 0.13 & $23 \rightarrow 24-24 \rightarrow 25$ & 0.68 & 0.38 & 0.14 & 0.00 \\
\hline $03 \rightarrow 04-04 \rightarrow 05$ & 0.00 & 0.83 & 0.24 & 0.24 & $24 \rightarrow 25-25 \rightarrow 26$ & 0.00 & 0.95 & 0.85 & 0.01 \\
$04 \rightarrow 05-05 \rightarrow 06$ & 0.71 & 0.45 & 0.62 & 0.11 & $25 \rightarrow 26-26 \rightarrow 27$ & 0.01 & 0.34 & 0.88 & 0.01 \\
$05 \rightarrow 06-06 \rightarrow 07$ & 0.00 & 0.53 & 0.74 & 0.95 & $26 \rightarrow 27-27 \rightarrow 28$ & 0.00 & & 0.42 & 0.00 \\
$06 \rightarrow 07-07 \rightarrow 08$ & 0.00 & 0.50 & 0.80 & 0.25 & $27 \rightarrow 28-28 \rightarrow 29$ & 0.00 & & 0.84 & 0.95 \\
$07 \rightarrow 08-08 \rightarrow 09$ & 0.73 & 0.66 & 0.99 & 0.93 & $28 \rightarrow 29-29 \rightarrow 30$ & 0.03 & & 0.79 & 0.09 \\
$08 \rightarrow 09-09 \rightarrow 10$ & 0.00 & 0.75 & 0.88 & 0.11 & $29 \rightarrow 30-30 \rightarrow 31$ & 0.00 & & 0.73 & 0.13 \\
\hline $09 \rightarrow 10-10 \rightarrow 11$ & 0.33 & 0.00 & 0.27 & 0.94 & $30 \rightarrow 31-31 \rightarrow 32$ & 0.09 & & 0.25 & \\
$10 \rightarrow 11-11 \rightarrow 12$ & 0.00 & 0.00 & 0.00 & 0.06 & $31 \rightarrow 32-32 \rightarrow 33$ & 0.07 & & 0.45 & \\
$11 \rightarrow 12-12 \rightarrow 13$ & 0.18 & 0.91 & 0.00 & 0.16 & $32 \rightarrow 33-33 \rightarrow 34$ & 0.00 & & 0.85 & \\
\hline $12 \rightarrow 13-13 \rightarrow 14$ & 0.54 & 0.79 & 0.00 & 0.88 & $33 \rightarrow 34-34 \rightarrow 35$ & 0.00 & & 0.52 & \\
$13 \rightarrow 14-14 \rightarrow 15$ & 0.07 & 0.08 & 0.00 & 0.54 & $34 \rightarrow 35-35 \rightarrow 36$ & 0.36 & & 0.01 & \\
$14 \rightarrow 15-15 \rightarrow 16$ & 0.00 & 0.05 & 0.00 & 0.68 & $35 \rightarrow 36-36 \rightarrow 37$ & 0.21 & & 0.09 & \\
$15 \rightarrow 16-16 \rightarrow 17$ & 0.52 & 0.82 & 0.62 & 0.35 & $36 \rightarrow 37-37 \rightarrow 38$ & 0.69 & & 0.76 & \\
$16 \rightarrow 17-17 \rightarrow 18$ & 0.77 & 0.22 & 0.14 & 0.20 & $37 \rightarrow 38-38 \rightarrow 39$ & 0.75 & & 0.05 & \\
$17 \rightarrow 18-18 \rightarrow 19$ & 0.26 & 0.37 & 0.69 & 0.04 & $38 \rightarrow 39-39 \rightarrow 40$ & 0.01 & & 0.18 & \\
$18 \rightarrow 19-19 \rightarrow 20$ & 0.07 & 0.46 & 0.01 & 0.02 & $39 \rightarrow 40-40 \rightarrow 41$ & 0.02 & & 0.94 & \\
$19 \rightarrow 20-20 \rightarrow 21$ & 0.00 & 0.01 & 0.28 & 0.29 & $40 \rightarrow 41-41 \rightarrow 42$ & 0.01 & & 0.37 & \\
$20 \rightarrow 21-21 \rightarrow 22$ & 0.04 & 0.42 & 0.63 & 0.85 & $41 \rightarrow 42-42 \rightarrow 43$ & 0.78 & & 0.56 & \\
\hline
\end{tabular}

We now consider the focus, i.e., the difference of the changes of choice $\mathrm{B} \rightarrow \mathrm{A}-\mathrm{A} \rightarrow \mathrm{B}$ that gives us the tendency to converge toward the initial choice-if positive-or to move away from it-if negative. The results can be seen in Figure 10 and Table 8.

\subsection{Evolution of Non-Casuality, Flux, and Focus in Time}

In Figure 11, we show the evolution of the percentage of A's choice in chronological order for the four groups.

In Figure 12, we show the statistically significant differences between sessions for the percentage of A's choice and the percentage of $A \rightarrow B$ and $B \rightarrow A$ transitions in chronological order for the four groups. For the latter two, we use the notation explained in the section "Evolution of the transitions between questionnaires."

Considering the choice of $\mathrm{A}$, we show the statistically significant comparisons between consecutive sessions, including the questionnaire zero completed before the beginning of the training. For the transition $A \rightarrow B$ and $B \rightarrow A$, we use the 
Parents B->A-A->B (Focus) G1

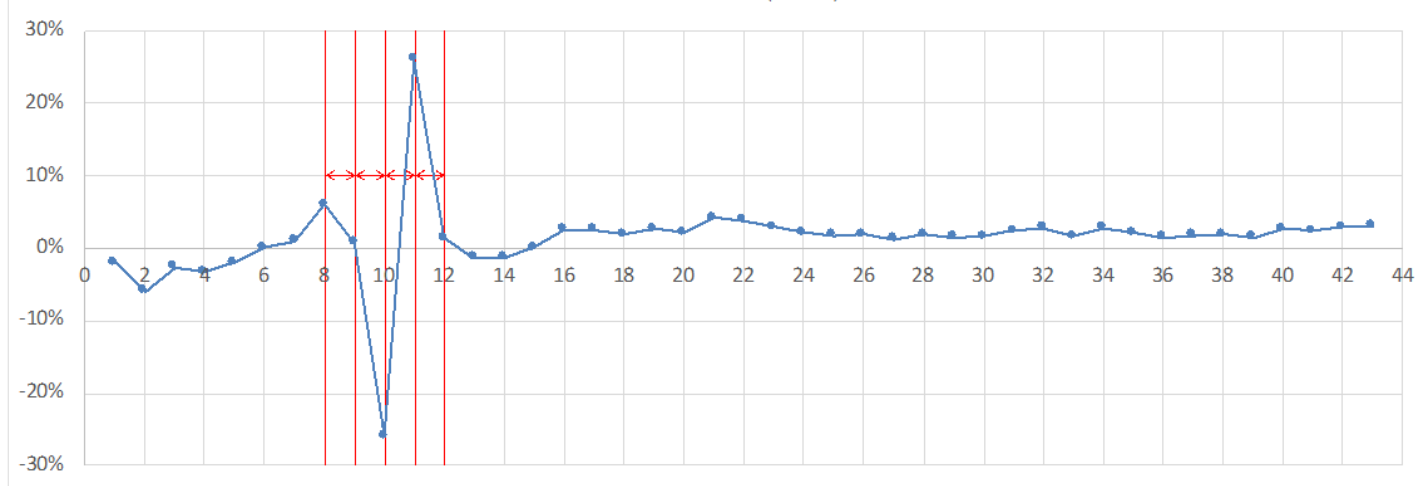

Parents B->A-A->B (Focus) G2

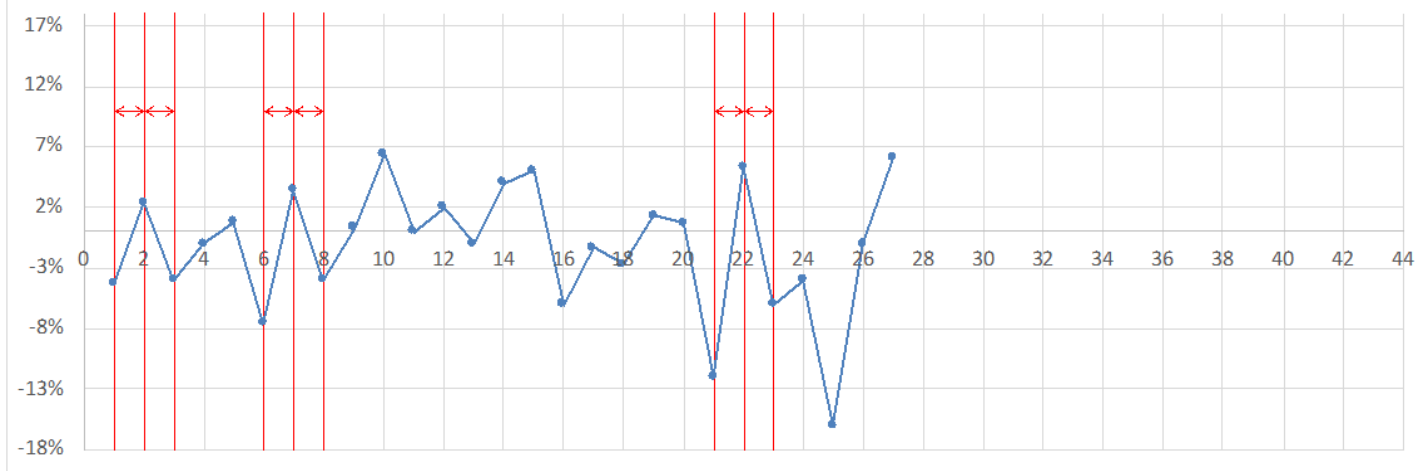

Young B->A-A->B (Focus) G1
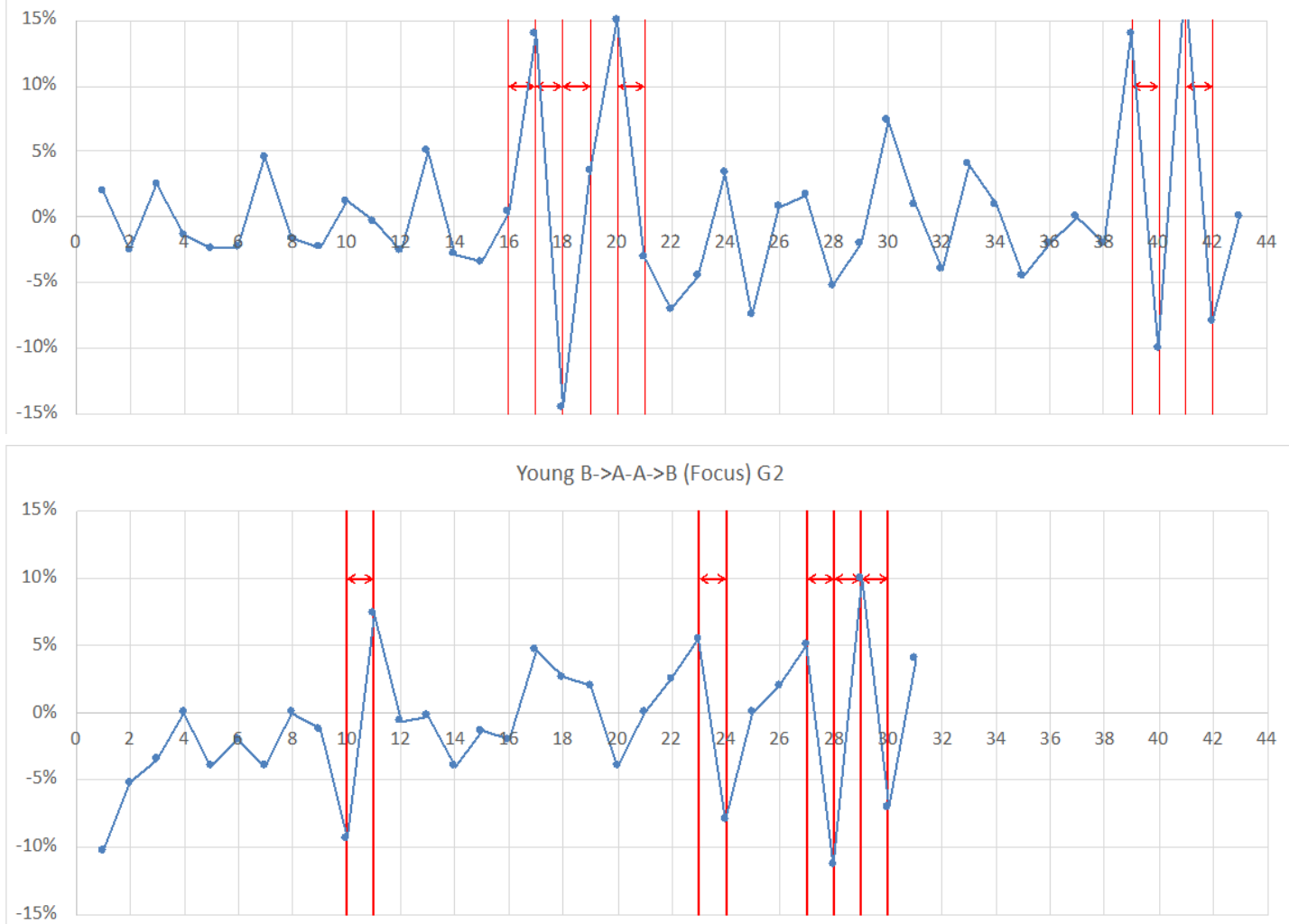

Figure 10. Comparison of the percentage of the $B \rightarrow A-A \rightarrow B$ changes between successive sessions for each group as calculated with the Conover test following a Friedmann test on the totality of the percentages. 


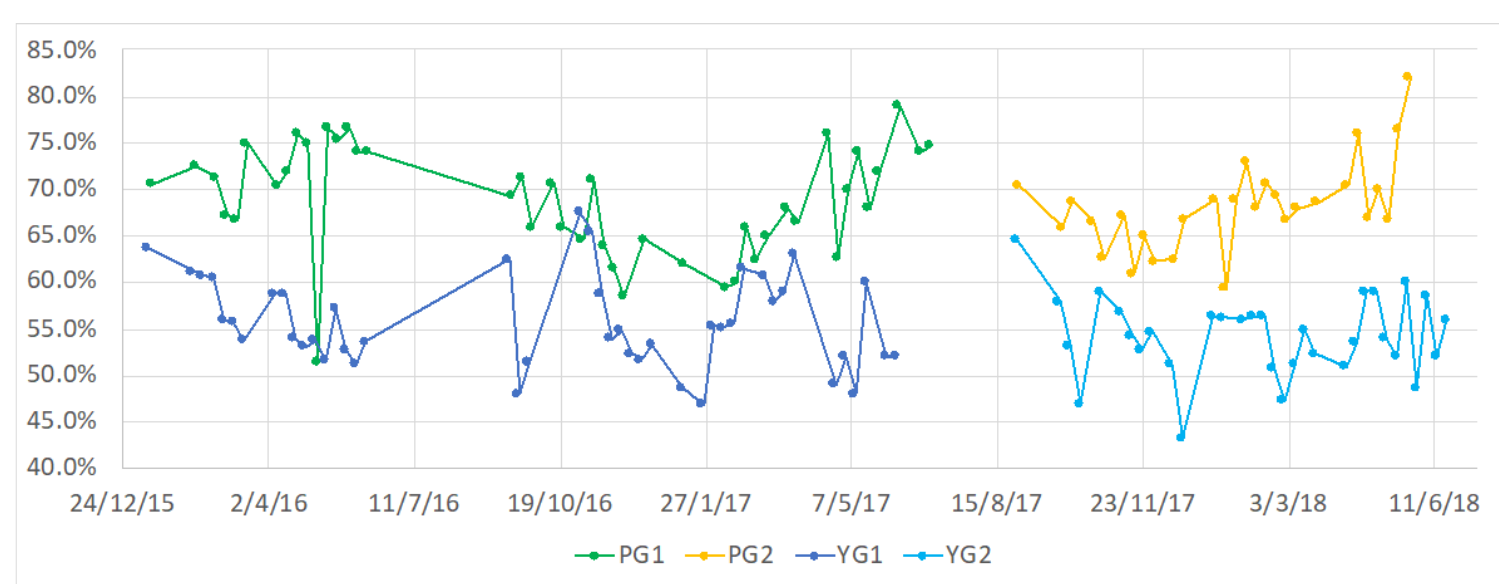

Figure 11. Evolution of the percentage of A's choice in chronological order for the four groups.

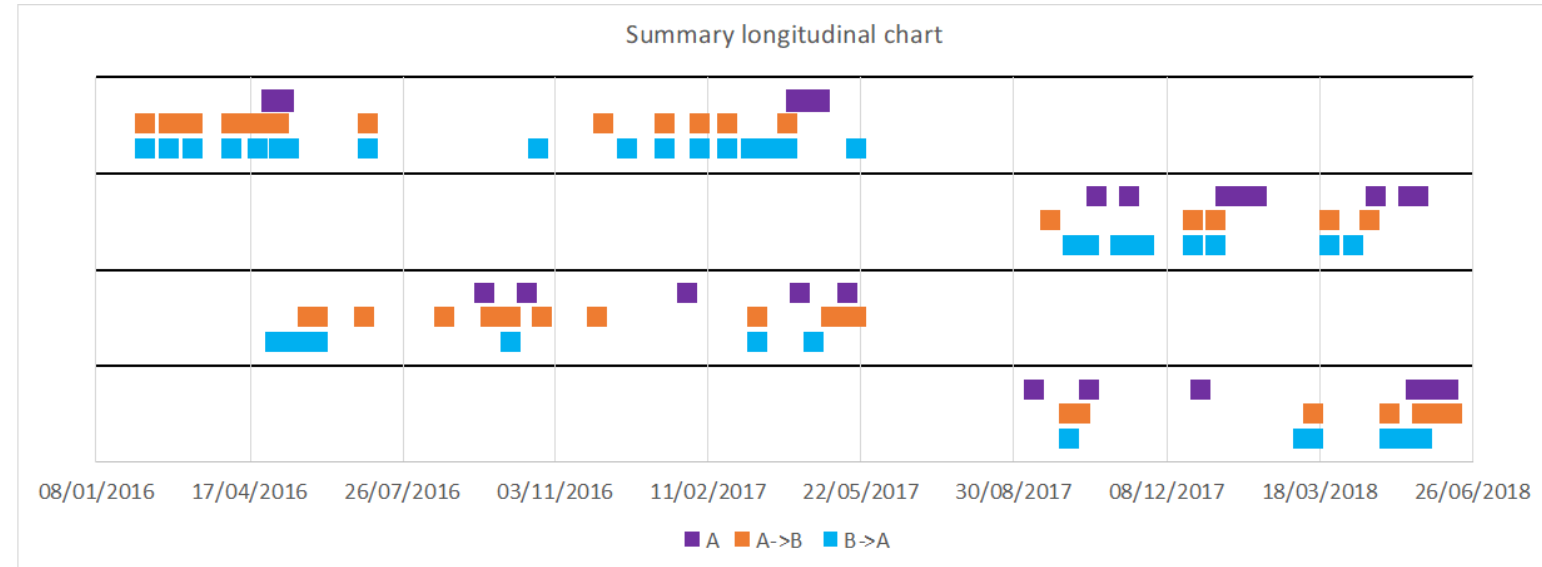

Figure 12. Statistically significant differences between sessions for the percentage of A's choice and the percentage of $\mathrm{A} \rightarrow \mathrm{B}$ and $\mathrm{B} \rightarrow \mathrm{A}$ transitions in chronological order for the four groups.

notation explained in the section "Evolution of the transitions between questionnaires."

In Figure 13, we show the statistically significant differences between sessions for the percentages of focus $(B \rightarrow A-A \rightarrow B)$, flux $(A \rightarrow B+B \rightarrow A)$, and non-casuality $(A \rightarrow B$ vs. $B \rightarrow A$ ) transitions in chronological order for the four groups. This allows us to determine whether there is a correlation between non-casuality, flux, and focus during the four groups' chronological evolution. We remember that we have explained the notation used for the questionnaire number in the section "Evolution of the transitions between questionnaires."

\section{Discussion}

\subsection{Theoretical Framework}

We believe that the best way to study the unconscious is to observe its amplification in group dynamics. As it happens in quantum mechanics, the problem of measurement is central also in psychophysics because the unconscious is, by definition, unknowledgeable and not only because it is "unconscious" but also 
Table 8. Comparison of the focus of choices $\mathrm{B} \rightarrow \mathrm{A}-\mathrm{A} \rightarrow \mathrm{B}$ between successive sessions with the Conover post-hoc test. Two changes of choices are statistically different when $p<0.05$, and the corresponding cell is red in the table.

\begin{tabular}{|c|c|c|c|c|c|c|c|c|c|}
\hline $\mathrm{B} \rightarrow \mathrm{A}-\mathrm{A} \rightarrow \mathrm{B}$ & PG1 & PG2 & YG1 & YG2 & $\mathrm{B} \rightarrow \mathrm{A}-\mathrm{A} \rightarrow \mathrm{B}$ & PG1 & PG2 & YG1 & YG2 \\
\hline $00 \rightarrow 01-01 \rightarrow 02$ & 0.68 & 0.02 & 0.42 & 0.09 & $21 \rightarrow 22-22 \rightarrow 23$ & 0.29 & 0.02 & 0.40 & 0.47 \\
\hline $01 \rightarrow 02-02 \rightarrow 03$ & 0.21 & 0.01 & 0.16 & 0.84 & $22 \rightarrow 23-23 \rightarrow 24$ & 0.54 & 0.88 & 0.26 & 0.01 \\
\hline $02 \rightarrow 03-03 \rightarrow 04$ & 0.19 & 0.28 & 0.24 & 0.50 & $23 \rightarrow 24-24 \rightarrow 25$ & 0.76 & 0.39 & 0.08 & 0.17 \\
\hline $03 \rightarrow 04-04 \rightarrow 05$ & 0.70 & 0.93 & 0.92 & 0.39 & $24 \rightarrow 25-25 \rightarrow 26$ & 0.74 & 0.09 & 0.19 & 0.76 \\
\hline $04 \rightarrow 05-05 \rightarrow 06$ & 0.76 & 0.11 & 0.80 & 0.70 & $25 \rightarrow 26-26 \rightarrow 27$ & 0.47 & 0.37 & 0.74 & 0.49 \\
\hline $05 \rightarrow 06-06 \rightarrow 07$ & 0.96 & 0.00 & 0.21 & 0.95 & $26 \rightarrow 27-27 \rightarrow 28$ & 0.45 & & 0.31 & 0.01 \\
\hline $06 \rightarrow 07-07 \rightarrow 08$ & 0.11 & 0.01 & 0.24 & 0.69 & $27 \rightarrow 28-28 \rightarrow 29$ & 0.72 & & 0.62 & 0.00 \\
\hline $07 \rightarrow 08-08 \rightarrow 09$ & 0.04 & 0.14 & 0.64 & 0.68 & $28 \rightarrow 29-29 \rightarrow 30$ & 0.95 & & 0.10 & 0.02 \\
\hline $08 \rightarrow 09-09 \rightarrow 10$ & 0.00 & 0.35 & 0.73 & 0.23 & $29 \rightarrow 30-30 \rightarrow 31$ & 0.51 & & 0.25 & 0.10 \\
\hline $09 \rightarrow 10-10 \rightarrow 11$ & 0.00 & 0.30 & 0.79 & 0.01 & $30 \rightarrow 31-31 \rightarrow 32$ & 0.57 & & 0.38 & \\
\hline $10 \rightarrow 11-11 \rightarrow 12$ & 0.00 & 0.80 & 0.63 & 0.36 & $31 \rightarrow 32-32 \rightarrow 33$ & 0.10 & & 0.25 & \\
\hline $11 \rightarrow 12-12 \rightarrow 13$ & 0.12 & 0.71 & 0.28 & 0.80 & $32 \rightarrow 33-33 \rightarrow 34$ & 0.12 & & 0.42 & \\
\hline $12 \rightarrow 13-13 \rightarrow 14$ & 0.83 & 0.56 & 0.26 & 0.50 & $33 \rightarrow 34-34 \rightarrow 35$ & 0.44 & & 0.55 & \\
\hline $13 \rightarrow 14-14 \rightarrow 15$ & 0.76 & 0.78 & 0.98 & 0.65 & $34 \rightarrow 35-35 \rightarrow 36$ & 0.51 & & 0.51 & \\
\hline $14 \rightarrow 15-15 \rightarrow 16$ & 0.09 & 0.12 & 0.50 & 0.88 & $35 \rightarrow 36-36 \rightarrow 37$ & 0.72 & & 0.81 & \\
\hline $15 \rightarrow 16-16 \rightarrow 17$ & 0.78 & 0.64 & 0.04 & 0.27 & $36 \rightarrow 37-37 \rightarrow 38$ & 0.91 & & 0.81 & \\
\hline $16 \rightarrow 17-17 \rightarrow 18$ & 0.41 & 0.72 & 0.00 & 0.67 & $37 \rightarrow 38-38 \rightarrow 39$ & 0.82 & & 0.13 & \\
\hline $17 \rightarrow 18-18 \rightarrow 19$ & 0.36 & 0.27 & 0.00 & 0.74 & $38 \rightarrow 39-39 \rightarrow 40$ & 0.23 & & 0.03 & \\
\hline $18 \rightarrow 19-19 \rightarrow 20$ & 0.45 & 0.35 & 0.06 & 0.19 & $39 \rightarrow 40-40 \rightarrow 41$ & 0.68 & & 0.01 & \\
\hline $19 \rightarrow 20-20 \rightarrow 21$ & 0.37 & 0.32 & 0.00 & 0.51 & $40 \rightarrow 41-41 \rightarrow 42$ & 0.59 & & 0.02 & \\
\hline $20 \rightarrow 21-21 \rightarrow 22$ & 0.46 & 0.01 & 0.33 & 0.70 & $41 \rightarrow 42-42 \rightarrow 43$ & 0.84 & & 0.47 & \\
\hline
\end{tabular}

Summary longitudinal chart

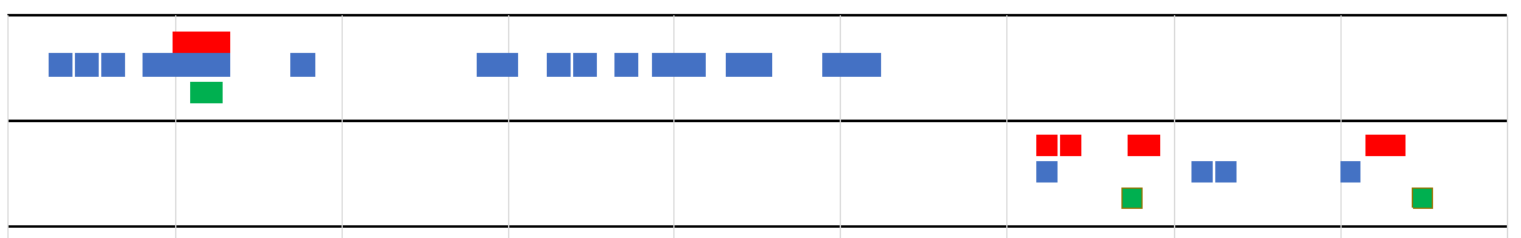

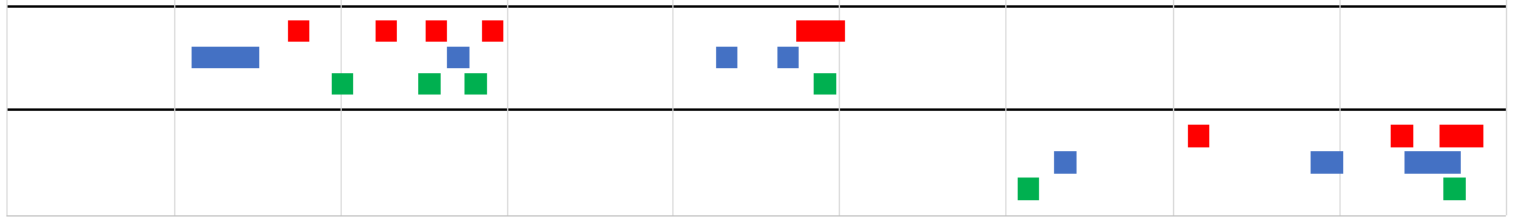

08/01/2016 17/04/2016 26/07/2016 $\quad 03 / 11 / 2016 \quad 11 / 02 / 2017 \quad 22 / 05 / 2017 \quad 30 / 08 / 2017 \quad 08 / 12 / 2017 \quad 18 / 03 / 2018 \quad 26 / 06 / 2018$

- Focus a Flux Non-Casuality

Figure 13. Statistically significant differences between sessions for the percentage of focus $(B \rightarrow A-A \rightarrow B)$, flux $(A \rightarrow B+B \rightarrow A)$, and non-casuality $(A \rightarrow B$ vs. $B \rightarrow A)$ transitions in chronological order for the four groups.

because the "detector" is the cognitive part of the individual, which is deeply influenced by, and indeed built upon, the unconscious.

This analogy relies on the observation that group dynamics, as described by 
Bion's "basic assumptions," is similar to individual dynamics, in particular in the crucial aspect of the analogy of the mourning process in the individual with the loss of the ideal leader in the group, inspired by the Oedipean constellation.

Considering the simultaneous presence of potentially connected individuals, we have made the hypothesis that the entanglement effects could be more pronounced in the case of a group setting. We call this "quantum amplification." As said in the Introduction, Jung (Jung \& McGuire, 1925) was aware that the individual psyche is tributary of a layered unconscious, where the layers are the "collective unconscious" of larger and larger groups, such as the family and society.

Jung also speaks of amplification (Jung \& Hull, 1911). For Jung, the "amplification" is the extension and the deepening of a dream-like image by means of associations centered on the dream theme and associations based upon social studies and the history of symbols (mythology, mysticism, folklore, religion, ethnology, art, etc.). Thanks to this, the dream becomes accessible to interpretation (Jung, 1962). If we consider the analogy conveyed by the term "amplification" in the two contexts, unconscious mental processes like dreams can be regarded as "microscopic" quantum processes, becoming accessible to conscience only via an amplification and measurement process, in this case, operated by consciousness. This is one more example of the interesting parallels that we can draw between quantum physics and psychodynamics.

In the case presented in this article, we can suppose that the group situation generated by the DBT skills training is influenced not only by the individual and family situations but also by the environment, such as the large social group.

In quantum physics, a microscopic process is "amplified" by the "observer" to the macroscopic level, allowing the measurement to take place. Thus, only after amplification, we can observe a microscopic quantum process as a physical phenomenon via an act of measurement. However, the irreversibility of such an act is still an open argument of discussion.

The perspective of this study is to observe if the group dynamics-particularly in a slow-open setting-is also affected by the environment (as in quantum mechanics).

In the present experiment, we noted some differences between parents and adolescents-for example, in the numbers of sessions, similar to what we have observed in one of our previous work of ours (Fernandez-Rivas et al., 2020) —but the comparison between parents and adolescents groups will be the subject of a future study.

\subsection{Percentage of A's Answers}

The most striking aspect of our data is that the answers to the questionnaire zero, administered before the training, are very skewed, with values of A's percentages respectively of $70.6 \%$ for PG1, 70.5\% for PG2, $63.7 \%$ for YG1, 64.7\% for YG2. This is similar to what we found in a previous study of DBT closed groups 
(Fernandez-Rivas et al., 2020; Trojaola-Zapirain et al., 2019) at the end of the training.

We observed an increase to $74.7 \%$ for PG1 and to $82 \%$ for PG2, and a reduction to $52 \%$ for YG1 and 56\% for YG2.

The trends in the changes of the A's answers are different in the four groups, and it is interesting to note that both groups of parents, PG1 and PG2, show an increase in the choice of the initial preferred image $(+4.1 \%$ and $+11.5 \%)$ while both groups of young YG1 and YG2 show a decrease $(-11.7 \%$ and $-5.6 \%)$.

\subsection{Analysis of "Flux" and "Focus" of the Answers}

The changes $A \rightarrow B$ and $B \rightarrow A$ in each of the four groups are similar, and this is confirmed that there are few non-casuality significant differences. We recall that we indicate as non-casuality the fact that the changes $A \rightarrow B$ and from $B \rightarrow A$ are not in equilibrium, i.e., the percentage of changes of the 50 questions are not statistically comparable for the two directions.

The flux, being the sum of $A \rightarrow B$ and $B \rightarrow A$, presents statistically significant values almost at the same moment as $\mathrm{A} \rightarrow \mathrm{B}$ and $\mathrm{B} \rightarrow \mathrm{A}$. On the other hand, the statistically significant values for the focus are less than in $A \rightarrow B, B \rightarrow A$, and flux.

The interpretation of this observation is not simple: if we imagine that the originally preferred answer-the A answer of the questionnaire zero-is the "personal" answer independent from the influence of the group, we can suppose that the return to this choice signals a movement "against" the "group continuum" and toward loyalty to the familiar and social clan that we can define as "normal clanity."

The PG1 group shows many significant variations of the flux (but also of $A \rightarrow B$ and $B \rightarrow A$ ) between different sessions, more than in the other groups. We note that focus and non-casuality present significative differences in the same phase of the training, i.e., before the $20^{\text {th }}$ session and at the end of the training (except for PG1). This is consistent with the "canonical" group evolution. At the beginning of the training, the group experiences the groupal disillusion. At the end, the group reacts against and mourns the conclusion of the experience, and participants tend to return to their normal clanity to preserve themselves from the group's disillusion/demise.

\subsection{Longitudinal Analysis}

Because the groups had a training and therapeutical goal, the fact that some groups took place simultaneously was casual and independent from the research objectives. Nevertheless, this overlap in time of the different groups allows a comparison of their evolution in time. Probably because of the slow-open structure of the groups, we observed some coincidence in their evolution which could depend more on external factors than on the group's internal evolution.

If we observe the group evolution in calendar time rather than in the number of sessions, we see the same coincidences in the statistically significant values of 
some of the parameters considered ( $A, A \rightarrow B, B \rightarrow A$, flux), which seem to obey not only the internal evolution of the groups but also other possible external factors. This coincidence was not present for non-casuality and focus.

We underline that if the flux is the expression of changes from $A$ to $B$ plus $B$ to $A$, the non-casuality indicates the statistical difference of the transitions $A \rightarrow B$ and $B \rightarrow A$, and the focus, defined as $B \rightarrow A$ minus $A \rightarrow B$, is the expression of the tendency to revert to the initial choice of pictures before the beginning of the training.

The interaction with the external environment in slow-open groups is probably experienced by the participants in an interactive mode with important clan loyalties: the high "orientation" (A's choices before the constitution of the group) is "recovered" and in a group even exceeded during the sessions and the answers are less casual (less 50\%) than in a state of "group continuum." We note that the participants remain in the group for more or less than $10-12$ sessions. Therefore, we can suppose that the slow-open group promotes a "clan continuum" due more to the external environment than a group continuum.

\section{Conclusion}

We conducted this experiment in 2017-2019 during Dialectical Behavior Therapy skills training at the Psychiatric Service of Basurto University Hospital in Bilbao, Spain. Results of similar experiments have been reported in previous publications (Fernandez-Rivas et al., 2020; Trojaola-Zapirain et al., 2014, 2015, 2016, 2019). The main conclusion of these studies was that the data were suggesting evidence in favor of the building of a group unconscious following Bion's "basic assumptions," where a strong interaction between the psyches of the group participants is established at the very beginning of the group experience, and then it slowly evolves under the known group dynamics. To describe this phenomenon, Bion has introduced the concept of "valency," indicating the immediacy of the basic assumptions' onset, more analogous to tropisms than to purposive behavior. This effect is enhanced in the group setting by an amplification process whereby groups "amplify emotional reactions, resulting in a combustible process of emotional contagion" (Bion, 1961).

The difficulty of verifying any theory of the unconscious is that we have no way to perform a direct measurement (Atmanspacher, 2004; Cerf \& Adami, 1997, 1998). To circumvent this problem, we have devised an indirect measure based on a questionnaire to be answered by the participants in a group situation.

It is interesting to note that such an experiment is trying to determine whether a psychic situation-the supposed entanglement of the individuals' unconscious in a group situation-has an actual effect on the material world-the answers provided to a questionnaire.

As we said above, the interpersonal relations in the group situation may have a relaxing effect on the neurotic attitudes with a consequent recovery of otherwise non-accessible psychical energy. Otherwise, particularly for adolescents, the 
feeling of identity loss could bring stress and anxiety and ultimately provoke the exit from the group when the "orientation" (A's choices) is minimal, and the confusion is highest. Psychoanalytically, we would call this a resistance to change. Conflicts in clan loyalties, common in adolescents when moving from the family envelope to the friends one may create a strong discomfort and lead to a flight from the group situation. The loyalty to the clan is more stable for the parents, and the return to the choice of the initially preferred image is clearly present. For the young participants, the groupal experience is more influencing and the return to the initially chosen image less marked. The group continuum comes from the entanglement of individual psyches toward forming a group unity having its own identity, in this case, a group identity. We can suppose that in slow-open groups, this entanglement is weaker than in closed groups. In slow-open groups, the influence of the external environment seems to be more important, and the conflict between friend clan, family clan, and group clan more manageable.

It might be interesting to attempt to derive some social and practical implications based on the research results, although this is not the primary purpose of the present work. Our study confirms that the slow open setting is the most adapted for the adolescents because it minimizes the adverse effects of the dropouts typical for this population. While there is a substantial turnover of participants, and the group population oscillates considerably_at least from the perspective of our data - the conflict between clanic loyalties (families and peers, e.g., friends) and the DBT group seems manageable, and the dropouts limited. This could suggest that the slow open setting plays an important role in creating an effective training and therapeutic environment for a population that is demanding but also challenging to treat consistently.

The participation in the Dialectical Behavior Group Therapy is probably reinforced by the slow-open setting, allowing adolescents and parents to experience a moderate conflict with clanic preexistent loyalties and a more relaxed interaction with group continuum.

\section{Conflicts of Interest}

The authors declare no conflicts of interest regarding the publication of this paper.

\section{References}

Aspect, A., Grangier, P., \& Roger, G. (1982). Experimental Realization of Einstein-Podolsky-Rosen-Bohm Gedankenexperiment: A New Violation of Bell's Inequalities. Physical Review Letters, 49, 91-94. https://doi.org/10.1103/PhysRevLett.49.91

Atmanspacher, H. (2004). Quantum Approaches to Consciousness. In Stanford Encyclopedia of Philosophy. Stanford University. https://plato.stanford.edu/entries/qt-consciousness

Atmanspacher, H., \& Fach, W. (2013). A Structural-Phenomenological Typology of MindMatter Correlations. Journal of Analytical Psychology, 58, 219-244.

https://doi.org/10.1111/1468-5922.12005 
Atmanspacher, H., Römer, H., \& Walach, H. (2002). Weak Quantum Theory: Complementarity and Entanglement in Physics and Beyond. Foundations of Physics, 32, 379406. https://doi.org/10.1023/A:1014809312397

Baaquie, B. E., \& Martin, F. (2005). Quantum Psyche-Quantum Field Theory of the Human Psyche. NeuroQuantology, 3, 7-42. https://doi.org/10.14704/nq.2005.3.1.57

Beck, F., \& Eccles, J. (1992). Quantum Aspects of Brain Activity and the Role of Consciousness. Proceedings of the National Academy of Sciences of the United States of America, 89, 11357-11361. https://doi.org/10.1073/pnas.89.23.11357

Bell, J. (1964). On the Einstein Podolsky Rosen Paradox. Physics, 1, 195-200. https://doi.org/10.1103/PhysicsPhysiqueFizika.1.195

Bell, J. (1966). On the Problem of Hidden Variables in Quantum Mechanics. Reviews of Modern Physics, 38, 447. https://doi.org/10.1103/RevModPhys.38.447

Bion, W. (1961). Experiences in Groups and Other Papers. Tavistock Publications Ltd.

Bohr, N. (1935). Can Quantum-Mechanical Description of Physical Reality Be Considered Complete? Physical Review, 48, 969-702. https://doi.org/10.1103/PhysRev.48.696

Cerf, N., \& Adami, C. (1997). Quantum Mechanics of Measurement. arXiv:Quant-Ph/ $9605002 \mathrm{v} 2$

Cerf, N., \& Adami, C. (1998). What Information Theory Can Tell Us about Quantum Reality. arXiv:Quant-Ph/9806047v1

Conover, W. J. (1999). Practical Nonparametric Statistics (3rd ed.). Wiley.

Conover, W. J., \& Iman, R., L. (1979). Multiple-Comparisons Procedures. Tech. Rep. LA7677-MS, Los Alamos Scientific Laboratory. https://doi.org/10.2172/6057803

Conte, E., Todarello, O., Federici, A., Vitiello, F., Lopane, M., \& Khrennikov, A. A. (2003). Preliminary Evidence of Quantum Like Behavior in Measurement of Mental State. arXiv:quant-ph/0307201v1

Einstein, A., Podolsky, B., \& Rosen, N. (1935). Can Quantum-Mechanical Description of Physical Reality Be Considered Complete? Physical Review, 47, 777-780. https://doi.org/10.1103/PhysRev.47.777

Fach, W. (2011). Phenomenological Aspects of Complementarity and Entanglement in Exceptional Human Experiences (ExE). Axiomathes, 21, 233-247. https://doi.org/10.1007/s10516-010-9143-7

Fernandez-Rivas, A., Sesma-Pardo, E., Kerexeta, I., Diaz-Cosgaya, A., Vivanco, E., Carminati, F., Torres, M. A. G., Fouassier, C., Martin, F., Demongeot, J., \& Carminati, G. G. (2020). Group Therapy for Adolescents and Parents: Study of Unconscious Orientation. NeuroQuantology, 18, 70-82. https://doi.org/10.14704/nq.2020.18.7.NQ20195

Foulkes, S. H. (1964). Therapeutic Group Analysis. International Universities Press.

Freeman, W., \& Vitiello, G. (2016). Matter and Mind Are Entangled in Two Streams of Images Guiding Behavior and Informing the Subject through Awareness. Mind and Matter, 14, 7-24.

Galli Carminati, G., \& Carminati, F. (2006). The Mechanism of Mourning: An Anti-Entropic Mechanism. NeuroQuantology, 4, 186-197. https://doi.org/10.14704/nq.2006.4.2.97

Galli Carminati, G., \& Martin, F. (2008). Quantum Mechanics and the Psyche. Physics of Particles and Nuclei, 39, 560-577. https://doi.org/10.1134/S1063779608040047

Galli Carminati, G., Martin, F., \& Carminati, F. (2017). A Very Simple Quantum Model of Mind and Matter. NeuroQuantology, 15, 186-199.

https://doi.org/10.14704/nq.2017.15.2.1031 
Grinberg-Zylberbaum, J., Delaflor, M., Attie, L., \& Goswami, A. (1994). The EinsteinPodolsky-Rosen Paradox in the Brain: The Transferred Potential. Physics Essays, 7, 422-428.

Hamer, R. M., \& Simpson, P. M. (2009). Last Observation Carried Forward versus Mixed Models in the Analysis of Psychiatric Clinical Trials. American Journal of Psychiatry, 166, 639-641. https://doi.org/10.1176/appi.ajp.2009.09040458

Hameroff, S., \& Penrose, R. (1996). Conscious Events as Orchestrated Spacetime Selections. Journal of Consciousness Studies, 3, 36-53.

Jung, C. G. (1952). Synchronicity: An Acausal Connecting Principle. Princeton University Press. https://doi.org/10.1515/9781400839162

Jung, C. G. (1959). The Archetypes and the Collective Unconscious (2nd ed., Vol. 9). Princeton University Press.

Jung, C. G. (1960). The Structure and Dynamics of the Psyche (Nachdr.). Routledge.

Jung, C. G. (1962). Memories and Dreams, Reflections. Vintage Editions.

Jung, C. G., \& Hull, R. F. C. (1911). Symbols of Transformation: An Analysis of the Prelude to a Case of Schizophrenia. Princeton University Press.

Jung, C. G., \& McGuire, W. (1925). Analytical Psychology: Notes of the Seminar Given in 1925. Princeton University Press.

http://liverpool.idm.oclc.org/login?url=https://www.jstor.org/stable/10.2307/j.ctt7rgpf https://doi.org/10.1515/9781400843077

Jung, C. G., Pauli, W., \& Meyer, C. A. (2001). Atom and Archetype: The Pauli/Jung Letters 1932-1958. Princeton University Press.

Kaës, R. (2010). Le sujet, le lien et le groupe. Groupalité psychique et alliances inconscientes. Cahiers de Psychologie Clinique, 1, 13-40. https://doi.org/10.3917/cpc.034.0013

Khrennikov, A. (2015). Quantum-Like Model of Unconscious-Conscious Dynamics. Frontiers in Psychology, 6, 997. https://doi.org/10.3389/fpsyg.2015.00997

Linehan, M. (1993). Cognitive-Behavioral Treatment of Borderline Personality Disorder. Guilford Press.

Linehan, M. (2015). DBT Skills Training Manual(2nd ed.). Guilford Press.

Marshall, I. (1989). Consciousness and Bose-Einstein Condensates. New Ideas in Psychology, 7, 73-83. https://doi.org/10.1016/0732-118X(89)90038-X

Martin, F., \& Galli Carminati, G. (2009). Synchronicity, Quantum Mechanics, and Psyche. In H. Atmanspacher, \& H. Primas (Eds.), Recasting Reality: Wolfgang Pauli's Philosophical Ideas and Contemporary Science (pp. 227-243). Springer-Verlag. https://doi.org/10.1007/978-3-540-85198-1 11

Martin, F., Carminati, F., \& Galli Carminati, G. (2009). Synchronicity, Quantum Information, and the Psyche. Journal of Cosmology, 3, 580-589.

Martin, F., Carminati, F., \& Galli Carminati, G. (2010). Quantum Information, Oscillations and the Psyche. Physics of Particles and Nuclei, 41, 425-451.

https://doi.org/10.1134/S1063779610030032

Martin, F., Carminati, F., \& Galli Carminati, G. (2013). Quantum Information Theory Applied to Unconscious and Consciousness. NeuroQuantology, 11, 16-33.

https://doi.org/10.14704/nq.2013.11.1.628

Miller, A. L., Rathus, J. H., \& Linehan, M. (2017). Dialectical Behavior Therapy with Suicidal Adolescents. Guilford Press.

Orlov, Y. F. (1982). The Wave Logic of Consciousness: A Hypothesis. International Journal of Theoretical Physics, 21, 37-53. https://doi.org/10.1007/BF01880263 
Penrose, R. (1994). Shadows of the Mind. Oxford University Press.

Penrose, R., \& Gardner, M. (1989). The Emperor's New Mind: Concerning Computers, Minds, and The Laws of Physics. Oxford University Press. https://doi.org/10.1093/oso/9780198519737.001.0001

Pitkänen, M. (1998). Quantum Mind. Quantum Mind Archives, 26.

Pitkänen, M. (2012). Quantum Mind in TGD Universe. Journal of Nonlocality, 1, 1-42. https://journals.sfu.ca/jnonlocality/index.php/jnonlocality/article/download/17/17

R Core Team (2020). R: A Language and Environment for Statistical Computing. R Foundation for Statistical Computing. https://www.R-project.org/

Richens, J. F., Selby, J. H., \& Al-Safi, S. W. (2017). Entanglement Is Necessary for Emergent Classicality in All Physical Theories. Physical Review Letters, 119, Article ID: 080503. https://doi.org/10.1103/PhysRevLett.119.080503

Sabbadini, S., \& Vitiello, G. (2019). Entanglement and Phase-Mediated Correlations in Quantum Field Theory. Application to Brain-Mind States. Applied Sciences, 9, 3203. https://doi.org/10.3390/app9153203

Schrödinger, E. (1935). Discussion of Probability Relations between Separated Systems. Mathematical Proceedings of the Cambridge Philosophical Society, 31, 555-563. https://doi.org/10.1017/S0305004100013554

Schrödinger, E. (1936). Probability Relations between Separated Systems. Mathematical Proceedings of the Cambridge Philosophical Society, 32, 446-452. https://doi.org/10.1017/S0305004100019137

Trojaola-Zapirain, B., Carminati, F., Fernandez Rivas, M. A., Gonzalez Torres, A., Gonzales de Mendivil, E., Fouassier, C., Martin, F., Demongeot, J., \& Galli Carminati, G. (2019). An Update and Generalization of Group Unconscious Orientation in OMIE Group Training for Therapists. Neuroquantology, 17, 14-30.

https://doi.org/10.14704/nq.2019.17.1.1918

Trojaola-Zapirain, B., Carminati, F., Gonzalez Torres, A., Gonzales de Mendivil, E., Fouassier, C., Gex-Fabry, M., Martin, F., Labarere, J., Demongeot, J., Lorincz, E. N., \& Galli Carminati, G. (2014). Group Unconscious Common Orientation: Exploratory Study at the Basque Foundation for the Investigation of Mental Health Group Training for Therapists. Neuroquantology, 12, 139-150. https://doi.org/10.14704/nq.2014.12.1.709

Trojaola-Zapirain, B., Carminati, F., Gonzalez Torres, A., Gonzales de Mendivil, E., Fouassier, C., Gex-Fabry, M., Martin, F., Labarere, J., Demongeot, J., Lorincz, E. N., \& Galli Carminati, G. (2015). Addendum on Entropy to the Exploratory Study on Group Unconscious at the Basque Foundation for the Investigation of Mental Health Group Training for Therapists. Neuroquantology, 13, 49-56.

https://doi.org/10.14704/nq.2015.13.1.780

Trojaola-Zapirain, B., Carminati, F., Gonzalez Torres, A., Gonzales de Mendivil, E., Fouassier, C., Gex-Fabry, M., Martin, F., Labarere, J., Demongeot, J., Lorincz, E. N., \& Galli Carminati, G. (2016). A Comparison of the Evolution and Entropy of Responses to Picture Choices on an "Absurdum Questionnaire” between Members of Two Different Training Groups. Neuroquantology, 14, 501-513.

https://doi.org/10.14704/nq.2016.14.3.919

Vergopoulos, T. (1983). La sensibilisation à la dynamique de groupe d'aprés W. R. Bion et S.H. Foulkes. Médecine et Hygiène, 1, 3149-3155.

Vitiello, G. (2003). Quantum Dissipation and Information: A Route to Consciousness Modelling. NeuroQuantology, 1, 266-279. https://doi.org/10.14704/nq.2003.1.2.15

Walach, H., \& Römer, H. (2011). Generalized Entanglement-A Nonreductive Option for 
a Phenomenologically Dualist and Ontologically Monist View of Consciousness. In H. Walach, S. Schmidt, \& W. B. Jonas (Eds.), Neuroscience, Consciousness and Spirituality (Vol. 1, pp. 81-95). Springer Netherlands.

https://doi.org/10.1007/978-94-007-2079-4 6

Zanello, A., Rouget-Weber, B., Gex Fabry, M. G., Maercker, A., \& Guimon, J. (2004). New Instrument to Assess Social Functioning in Mental Health Settings. European Journal of Psychiatry, 18, 76-78.

Zurek, H. (1981). Pointer Basis of Quantum Apparatus: Into What Mixture Does the Wave Packet Collapse? Physical Review D, 24, 1516.

https://doi.org/10.1103/PhysRevD.24.1516 